\title{
LISA observations of supermassive black holes: Parameter estimation using full post-Newtonian inspiral waveforms
}

\author{
Miquel Trias ${ }^{1}$ and Alicia M. Sintes ${ }^{1,2}$ \\ ${ }^{1}$ Departament de Física, Universitat de les Illes Balears, Cra. Valldemossa Km. 7.5, E-07122 Palma de Mallorca, Spain \\ ${ }^{2}$ Max-Planck-Institut für Gravitationsphysik (Albert-Einstein-Institut), Am Mühlenberg 1, 14476 Golm, Germany
}

(Received 31 July 2007; published 17 January 2008)

\begin{abstract}
We study parameter estimation of supermassive black hole binary systems in the final stage of inspiral using the full post-Newtonian gravitational waveforms. We restrict our analysis to systems in circular orbit with negligible spins, in the mass range $10^{8} M_{\odot}-10^{5} M_{\odot}$, and compare the results with those arising from the commonly used restricted post-Newtonian approximation. The conclusions of this work are particularly important with regard to the astrophysical reach of future Laser Interferometer Space Antenna measurements. Our analysis clearly shows that modeling the inspiral with the full post-Newtonian waveform, not only extends the reach to higher mass systems, but also improves in general the parameter estimation. In particular, there are remarkable improvements in angular resolution and distance measurement for systems with a total mass higher than $5 \times 10^{6} M_{\odot}$, as well as a large improvement in the mass determination.
\end{abstract}

DOI: 10.1103/PhysRevD.77.024030

PACS numbers: 04.25.Nx, 04.80.Nn, 95.55.Ym, 97.60.Lf

\section{INTRODUCTION}

Supermassive black hole binary systems, in the mass range $10^{8} M_{\odot}-10^{5} M_{\odot}$, will be detectable by the Laser Interferometer Space Antenna (LISA) [1-3] throughout the entire Universe. Observations of gravitational waves from this class of sources are among its highest priority targets. By measuring these gravitational waves we will have detailed information regarding general relativity itself and the behavior of space-time [4-9], precision measurements of the Universe as a whole [10-13], the formation and growth of massive black holes in galaxy evolution $[11,14,15]$, and black hole astrophysics [16,17]. Detection of gravitational waves will provide complementary information to conventional astronomy.

Supermassive black hole binaries are long lived sources in the LISA band. The whole coalescence of a compact binary system is commonly divided into three phases: the adiabatic inspiral, the merger, and the ringdown. Most of the signal-to-noise ratio (SNR) accumulates during the last days prior to coalescence and during the merger phase, but one critically relies on long integration times to disentangle the source parameters, in particular, to resolve the source position in the sky and measure its luminosity distance [18]. This is due to the motion of LISA around the sun that breaks the degeneracy in the parameters. Thus, in this paper we restrict our attention to the inspiral phase since this is the most interesting for parameter estimation.

Because of the complexity of the problem, most analysis carried out so far to address how accurately LISA can measure the source parameters and the implications for astronomy and cosmology, have considered only the inspiral restricted post-Newtonian waveforms $[7,11-13,18-$ 23], where all amplitude corrections are discarded and only post-Newtonian (PN) contributions to the phase are taken into account. Thus, the restricted-PN waveform consists of just the dominant harmonic at twice the orbital phase. Only in a few cases, it has been reported the importance of including higher order PN terms to the amplitude and the phase [24-27]. In the context of ground-based detectors, it was found that the consequences of amplitude correction in the templates are considerable [28-30].

The main purpose of the present analysis is to investigate in detail the impact of full versus restricted-PN waveforms for parameter estimation, by exploring a vast parameter space, specifically in the context of LISA, extending previous results. The waveform we use is described by 11 parameters. Therefore extensive large-scale, CPUintensive Monte Carlo simulations have been required for such an exhaustive study.

The conclusions of this work are particularly important with regard to the astrophysical reach of future LISA measurements. Our analysis clearly shows that modeling the inspiral with the full post-Newtonian waveforms, as compared to the restricted-PN ones, not only extends the reach to higher mass systems, up to $10^{8} M_{\odot}$, as discussed by Arun et al. [26], but also improves the parameter estimation. Improvements in angular resolution and distance measurement are remarkable for systems with a total mass higher than $5 \times 10^{6} M_{\odot}$. These results are in agreement with those recently found in [27].

The organization of this paper is as follows. In Sec. II, we discuss the LISA detector output, and we provide the total LISA noise curve employed in our analysis. In Sec. III we describe the gravitational wave signals from binary systems. Section IV reviews the basic concepts of signal parameter estimation in matched filtering. In Sec. V we spell out the assumptions about the waveform and the observations on which our analysis is based, we present a detailed description of the waveform model and all key steps to compute the SNR and the estimation of the parameter errors. Section VI presents the results of our 
investigations, where we compare the impact on parameter estimation of supermassive black hole binaries using full post-Newtonian inspiral waveforms versus the restricted ones. The results are presented for a given source location, exploring a vast parameter space, we also study how source location errors in terms of advanced warning times, and the errors dependency with redshift. Finally, Sec. VII concludes with a summary of the main results of this paper and present pointers to future work.

\section{LISA RESPONSE TO GRAVITATIONAL WAVES AND DETECTOR NOISE}

LISA consists of three spacecrafts arranged in an equilateral triangle orbiting the sun. The arms of the triangle are approximately $L=5 \times 10^{6} \mathrm{~km}$ in length, and the triangle is inclined at an angle of $60^{\circ}$ to the ecliptic. The entire triangular configuration spins as the antenna orbits the sun, rotating once during a single orbit. A gravitational wave interacting with the configuration causes the length of the three arms to oscillate.

For the supermassive black hole binary inspirals considered in this paper, most of the SNR accumulates at frequencies $f<10 \mathrm{mHz}$, so it is adequate to use the lowfrequency approximation to the LISA response function derived by Cutler [18]. In this approximation, LISA can be regarded as two independent gravitational wave detectors with $90^{\circ}$ arms and rotated $45^{\circ}$ with respect to one another.

The strain $h(t)$ produced at the output of the LISA Michelson interferometer by a gravitational wave (GW) signal characterized by two polarization states $h_{+}(t)$ and $h_{\times}(t)$ is

$$
h^{(i)}(t)=\frac{\sqrt{3}}{2}\left[F_{+}^{(i)}(t) h_{+}(t)+F_{\times}^{(i)}(t) h_{\times}(t)\right],
$$

where $F_{+}^{(i)}$ and $F_{\times}^{(i)}$ are the time-dependent antenna pattern functions, the factor $\sqrt{3} / 2$ comes from the $60^{\circ}$ opening angle of the LISA arms, and the $i=\mathrm{I}$, II labels the two independent Michelson outputs. The response functions $F_{+}^{(i)}(t)$ and $F_{\times}^{(i)}(t)$ depend on the direction and orientation of the source in the sky and they vary with time because during the observation, the interferometer changes orientation with respect to the source. We refer the reader to [18] for further discussions and details.

The total noise that affects the observation of radiation emitted by binary systems is given by the superposition of instrumental sources, $S_{n}^{\text {inst }}(f)$, and astrophysical foregrounds of unresolved radiation, $S_{n}^{\text {conf }}(f)$, the so-called confusion noise. The total noise spectral density $S_{n}(f)$ is therefore the sum of these two components

$$
S_{n}(f)=S_{n}^{\text {inst }}(f)+S_{n}^{\text {conf }}(f) .
$$

The noise contributions in each data stream I and II are by definition the same.
A good fit for the instrumental noise, for $f \leq 5 \mathrm{mHz}$, is given by [31,32]

$$
\begin{aligned}
S_{n}^{\text {inst }}(f)= & 6.12 \times 10^{-51} f^{-4}+1.06 \times 10^{-40} \\
& +6.12 \times 10^{-37} f^{2} \mathrm{~Hz}^{-1}
\end{aligned}
$$

where $f$ is in Hz. This is derived from the online sensitivity curve generator [33], which is based on the noise budgets specified in [1].

For the confusion noise we adopt the same analytical approximations given in [31], considering only noise from short-period galactic $S_{n}^{\mathrm{GWD}}$ and extragalactic binaries $S_{n}^{\mathrm{EWD}}$ (due to white dwarfs binaries), assuming they are all unresolvable, i.e., the worst case, and we ignore the effects of captures of compact objects. That is, we estimate the total effective noise density as

$$
\begin{aligned}
S_{n}^{\text {eff }}(f)= & \min \left\{\left[S_{n}^{\text {inst }}(f)+S_{n}^{\mathrm{EWD}}(f)\right]\right. \\
& \times \exp \left(\kappa T^{-1} d N / d f\right), S_{n}^{\text {inst }}(f)+S_{n}^{\mathrm{EWD}}(f) \\
& \left.+S_{n}^{\mathrm{GWD}}(f)\right\}
\end{aligned}
$$

where we take $\kappa T^{-1}=1.5 \mathrm{yr}^{-1}$,

$$
\begin{gathered}
\frac{d N}{d f}=2 \times 10^{-3} f^{-11 / 3} \mathrm{~Hz}^{-1}, \\
S_{n}^{\mathrm{GWD}}(f)=1.4 \times 10^{-44} f^{-7 / 3} \mathrm{~Hz}^{-1},
\end{gathered}
$$

and

$$
S_{n}^{\mathrm{EWD}}(f)=2.8 \times 10^{-46} f^{-7 / 3} \mathrm{~Hz}^{-1} .
$$

\section{BINARY BLACK HOLE COALESCENCE WAVEFORMS}

The coalescence of binary black holes is commonly divided into three successive epochs in the time domain: inspiral, merger, and ringdown. During the inspiral the distance between the black holes diminishes and the orbital frequency sweeps up. The waveforms are well modeled using the post-Newtonian approximation to general relativity. Eventually the post-Newtonian description of the orbit breaks down, and the black holes cannot be treated as point particles any more. What is more, it is expected that they will reach the innermost stable circular orbit (ISCO), at which the gradual inspiral ends and the black holes begin to plunge together to form a single black hole. This is referred as the merger phase. At the end, the final black hole will gradually settle down into a Kerr black hole.

In recent years, a series of breakthroughs has occurred in numerical simulations of binary black hole systems [3436]. Long-term evolutions of inspiralling black holes that last for several orbits have been obtained with several independent codes [37-46], and accurate GW signals have been computed, including the merger and ringdown phases. Still, the post-Newtonian approximation to general 
relativity is the best available method for calculating the vast majority of the GW signal cycles observed by LISA. See [47] for a review and extensive references.

For nonspinning black holes, the best PN waveforms currently available have been calculated at $2.5 \mathrm{PN}$ order in amplitude and 3.5PN order in phase [48-53]. These waveforms in the two polarizations $h_{+}$and $h_{\times}$take the general form

$$
\begin{aligned}
h_{+, \times}= & \frac{2 M \eta}{D_{L}}(M \omega)^{2 / 3}\left\{H_{+, \times}^{(0)}+v^{1 / 2} H_{+, \times}^{(1 / 2)}+v H_{+, \times}^{(1)}\right. \\
& \left.+v^{3 / 2} H_{+, \times}^{(3 / 2)}+v^{2} H_{+, \times}^{(2)}+v^{5 / 2} H_{+, \times}^{(5 / 2)}\right\},
\end{aligned}
$$

where we have set $G=c=1$, as we will do throughout this paper, $v \equiv(M \omega)^{2 / 3}, \omega$ is the orbital frequency, $D_{L}$ is the luminosity distance to the source, and $M$ and $\eta$ are the observed total mass and the symmetric mass ratio respectively, defined in Sec. V. The explicit expressions for $H_{+, \times}^{(m / 2)}, m=0, \ldots, 5$ can be found in [50,54]. They include contributions from several harmonics of the binary's orbital motion.

For black holes with significant spins, the state of the art is somehow less advanced and the corresponding waveforms have been calculated through $2.5 \mathrm{PN}$ order $[55,56]$.

Equation (3.1) corresponds to the so-called full waveform (FWF). Given its complexity, together with the fact that the second harmonic contributes most strongly to the waveform over most of the inspiral phase, it is common to make some simplifications and work only with the restricted waveform (RWF), in which one neglects all amplitude terms except the Newtonian quadrupole one, but keeping the phase to some specific PN order, i.e., keeping only $H_{+, \times}^{(0)}$ and throwing out the rest $H_{+, \times}^{(m / 2)}$ for $m>0$.

It is the goal of this paper to revisit the problem of parameter estimation for supermassive black hole binaries studying the improvement in error estimation by using FWF and compare with the previous results obtained with the RWF.

\section{REVIEW OF SIGNAL ANALYSIS AND PARAMETER ESTIMATION}

In this section we briefly review the basic concepts and formulas of signal parameter estimation relevant to the goal of this paper; we refer the reader to [57] for a more detailed analysis.

The signal $s^{(i)}(t)$ as measured by the detector $i$ is a superposition of noise $n^{(i)}(t)$ and gravitational waves $h^{(i)}(t ; \boldsymbol{\lambda})$

$$
s^{(i)}(t)=h^{(i)}(t ; \boldsymbol{\lambda})+n^{(i)}(t)
$$

where $\boldsymbol{\lambda}$ represents a vector of the unknown parameters (location, masses, spins, etc.) that characterize the actual waveform and that one wishes to estimate from the data stream.
For sake of simplicity we shall made the standard assumptions that the noise $n^{(i)}(t)$ has zero mean and it is stationary and Gaussian. Within this approximation, the Fourier components of the noise are statistically described by

$$
E\left[\tilde{n}(f) \tilde{n}^{*}\left(f^{\prime}\right)\right]=\frac{1}{2} \delta\left(f-f^{\prime}\right) S_{n}(f),
$$

where $E[]$ denotes the expectation value with respect to an ensemble of noise realization, the $*$ superscript denotes complex conjugate, $S_{n}(f)$ is the one-sided noise power spectral density, and tildes denote Fourier transforms according to the convention

$$
\tilde{x}(f)=\int_{-\infty}^{\infty} e^{i 2 \pi f t} x(t) d t
$$

With a given noise spectral density for the detector, one defines the "inner product" between any two signals $g(t)$ and $h(t)$ by

$$
(g \mid h) \equiv 2 \int_{0}^{\infty} \frac{\tilde{g}^{*}(f) \tilde{h}(f)+\tilde{g}(f) \tilde{h}^{*}(f)}{S_{n}(f)} d f .
$$

With this definition, the probability of the noise to have a realization $n_{0}$ is just

$$
p\left(n=n_{0}\right) \propto e^{-\left(n_{0} \mid n_{0}\right) / 2} .
$$

The optimal signal-to-noise ratio (SNR) $\rho$, achievable with linear methods (e.g., matched filtering the data) is given by the standard expression

$$
\rho^{2}=(h \mid h)=4 \int_{0}^{\infty} \frac{|\tilde{h}(f)|^{2}}{S_{n}(f)} d f .
$$

In the limit of large SNR, which is typically the case for LISA observations of supermassive black hole binary systems, the probability that the gravitational wave signal $h(t ; \boldsymbol{\lambda})$ is characterized by a given set of values of the source parameters $\boldsymbol{\lambda}=\left\{\lambda^{k}\right\}$ is given by a Gaussian probability of the form [58]

$$
p(\boldsymbol{\lambda} \mid h)=p^{(0)}(\boldsymbol{\lambda}) \exp \left[-\frac{1}{2} \Gamma_{j k} \Delta \lambda^{j} \Delta \lambda^{k}\right],
$$

where $\Delta \lambda^{k}$ is the difference between the true value of the parameter and the best-fit parameter in the presence of some realization of the noise, $p^{(0)}(\boldsymbol{\lambda})$ represents the distribution of prior information (a normalization constant) and $\Gamma_{j k}$ is the so-called Fisher information matrix defined by

$$
\begin{aligned}
\Gamma_{i j} & \equiv\left(\partial_{i} h \mid \partial_{j} h\right) \\
& =2 \int_{0}^{\infty} \frac{\partial_{i} \tilde{h}^{*}(f) \partial_{j} \tilde{h}(f)+\partial_{i} \tilde{h}(f) \partial_{j} \tilde{h}^{*}(f)}{S_{n}(f)} d f,
\end{aligned}
$$

where $\partial_{i}=\frac{\partial}{\partial \lambda^{i}}$.

The inverse of the Fisher matrix, known as the variancecovariance matrix, gives us the accuracy with which we 
expect to measure the parameters $\lambda^{k}$

$$
\sum^{j k} \equiv\left(\Gamma^{-1}\right)^{j k}=\left\langle\Delta \lambda^{j} \Delta \lambda^{k}\right\rangle .
$$

Here the angle brackets denote an average over the probability distribution function in Eq. (4.7). The root-meansquare error $\sigma_{k}$ in the estimation of the parameters $\lambda^{k}$ can then be calculated, in the limit of large SNR, by taking the square root of the diagonal elements of the variancecovariance matrix,

$$
\sigma_{k}=\left\langle\left(\Delta \lambda^{k}\right)^{2}\right\rangle^{1 / 2}=\sqrt{\Sigma^{k k}},
$$

and the correlation coefficients $c^{j k}$ between two parameters $\lambda^{j}$ and $\lambda^{k}$ are given by

$$
c^{j k}=\frac{\left\langle\Delta \lambda^{j} \Delta \lambda^{k}\right\rangle}{\sigma_{j} \sigma_{k}}=\frac{\Sigma^{j k}}{\sqrt{\sum^{j j} \Sigma^{k k}}} .
$$

Returning again to the two detector case, we have that the largest value of the SNR is

$$
\begin{gathered}
\rho^{\mathrm{tot}}=\sqrt{\left(\rho^{I}\right)^{2}+\left(\rho^{I I}\right)^{2}}, \\
\left(\rho^{I, I I}\right)^{2}=\left(h^{I, I I} \mid h^{I, I I}\right),
\end{gathered}
$$

and we can write a total Fisher matrix as the sum of the individual Fisher matrices for each detector

$$
\Gamma_{i j}^{\mathrm{tot}}=\Gamma_{i j}^{I}+\Gamma_{i j}^{I I} \text {. }
$$

In this paper we will use Eq. (4.9), along with the FWF and RWF models for the binary black hole coalescence, and the LISA noise spectrum discussed in Sec. II, in order to estimate how well LISA will be able to measure the source parameters. We refer the reader to $[59,60]$ for a detailed discussion about the conditions required for the Fisher-matrix formalism to be applicable.

\section{OBSERVATION OF SUPERMASSIVE BLACK HOLE BINARY SYSTEMS}

We consider observations of supermassive black hole binary systems of masses $m_{1}$ and $m_{2}$ at luminosity distance $D_{L}$. For later convenience we define the following mass parameters: total mass $M=m_{1}+m_{2}$, reduced mass $\mu=$ $m_{1} m_{2} / M$, the symmetric mass ratio $\eta=\mu / M$, and $\mathcal{M}=$ $\mu^{3 / 5} M^{2 / 5}=M \eta^{3 / 5}$ the chirp mass. We focus on binary systems in the mass range $10^{8} M_{\odot}-10^{5} M_{\odot}$ and we do not consider here the case of binaries with an extreme mass ratio, e.g, a black hole of $10 M_{\odot}$ orbiting a supermassive one. The reason is that some assumptions about the waveform that we will be considering would be rather unrealistic for such an astrophysical system.

Before presenting the results, in this section we spell out the assumptions about the waveform and the observations on which our analysis is based, together with a detailed description of the post-Newtonian inspiral waveform we use.

\section{A. Assumptions}

The waveform model we consider is based on the following assumptions:

(i) As signal we consider only the inspiral phase of the whole coalescence, neglecting all information coming from the merger and the ringdown phases. We terminate the inspiral when the binary's members are separated by a distance $6 M$; this very roughly corresponds to the point at which the post-Newtonian expansion ceases to be accurate.

(ii) We restrict ourselves to circular orbits by omitting the orbital eccentricity; this hypothesis is considered rather realistic for the supermassive binary systems visible in the LISA band we consider.

(iii) The contributions of spins are negligible or they are oriented in such a way that no spin-induced precession of the orbital plane takes place; we take care of spin contributions only into the waveform phase. We choose this hypothesis in order to control the complexity of the problem and focus on the comparison of the FWF versus RWF.

(iv) We approximate the waveform at the $2 \mathrm{PN}$ order, both in amplitude and in phase, considering up to six harmonics in the case of FWF. This simplification is motivated by computational reasons due to our limited computational resources and does not affect in any significant way the final results.

(v) Out of the 17 parameters on which the most general waveform depends on, the $2 \mathrm{PN}$ approximation we are considering here depends only on 11 parameters: the luminosity distance, four angles defining the constant source position and orientation of the binary in the orbital plane, two mass parameters, two parameters related to the spin-orbit and spin-spin coupling, and one arbitrary phase and time.

We assume that the observations are carried out according to the following:

(i) We consider sources at cosmological distances, as the event rate of massive black hole binary systems in our local Universe is likely to be negligible and only taking into account the whole Universe it becomes of significant importance; moreover there is a great interest in carrying high redshift surveys; indeed, unless differently stated, we consider fiducial sources to be at redshift $z=1$, in a flat universe described by the following cosmological parameters: $H_{0}=71 \mathrm{~km} \mathrm{~s}^{-1} \mathrm{Mpc}^{-1}, \Omega_{m}=0.27$, and $\Omega_{\Lambda}=$ 0.73 ; the corresponding luminosity distance is therefore

$$
D_{L}(z)=\frac{1+z}{H_{0}} \int_{0}^{z} \frac{d z^{\prime}}{\sqrt{\Omega_{m}\left(1+z^{\prime}\right)^{3}+\overline{\Omega_{\Lambda}}}} .
$$

All the parameters considered here are the observed ones; they differ from the values of the parameters as measured in the source rest frame according to 


$$
\begin{gathered}
f \rightarrow \frac{f}{1+z} \quad t \rightarrow(1+z) t \quad M \rightarrow(1+z) M \\
\mathcal{M} \rightarrow(1+z) \mathcal{M} \quad \mu \rightarrow(1+z) \mu .
\end{gathered}
$$

(ii) Unless differently stated, we consider that LISA observes the inspiral for a whole year before it reaches the ISCO. This corresponds to different frequency ranges depending on the harmonic. For every choice of source parameters these frequencies are computed in advanced and we also impose a low-frequency cutoff to the instrument at $5 \times$ $10^{-5} \mathrm{~Hz}$.

(iii) The total noise that affects the observation is given by Eq. (2.4), that is, we take both the instrumental and confusion noise contributions.

(iv) We compute the expected mean-square errors $\left\langle\left(\Delta \lambda^{k}\right)^{2}\right\rangle^{1 / 2}$ and the angular resolution of the instrument, which we define as

$$
\Delta \Omega_{N}=2 \pi \sqrt{\left\langle\Delta \cos \theta_{N}^{2}\right\rangle\left\langle\Delta \phi_{N}^{2}\right\rangle-\left\langle\Delta \cos \theta_{N} \Delta \phi_{N}\right\rangle^{2}},
$$

where $\left(\theta_{N}, \phi_{N}\right)$ are the polar angles in the solar system barycentre frame of the source location in the sky, with one and with both detectors, but we only report the results for the combined case.

(v) The analysis is done in the frequency domain using the stationary phase approximation: we first compute analytically the derivatives $\partial_{j} \tilde{h}^{(i)}$, where $j=$ $1, \ldots, 11$, then compute numerically the Fisher matrix $\Gamma_{i j}$ and the variance-covariance matrix $\Sigma^{j k}$; the integration and matrix inversion are performed using numerical routines of the gsl library.

(vi) To provide an overall picture of the instrument performances, we study not just a few cases in detail but we also do extensive Monte Carlo simulations, by varying the relevant source parameters, in particular, the position and orientation of the source, as it turns out to affect very significantly the parameter measurements.

\section{B. Post-Newtonian inspiral waveforms}

In this section we derive explicit ready-to-use analytical expressions for the signal measured at the LISA detector output for inspiral binary systems in circular orbit within the $2 \mathrm{PN}$ approximation, that could easily be expanded to include higher order terms.

For our analysis, it is convenient to expand $h^{(i)}(t)$ as a summation of different multipole terms, which can be written schematically as

$$
\begin{aligned}
h^{(i)}(t)= & \sum_{j=1}^{6} h_{j}^{(i)}(t)=\sum_{j=1}^{6} \frac{\sqrt{3}}{2} 2 M \eta \frac{1}{D_{L}} x^{2} \\
& \times\left[\left(u_{+, j} F_{+}+u_{\times, j} F_{\times}\right) \cos \left(\frac{j}{2} \Phi+\varphi_{D}\right)\right. \\
& \left.+\left(w_{+, j} F_{+}+w_{\times, j} F_{\times}\right) \sin \left(\frac{j}{2} \Phi+\varphi_{D}\right)\right],
\end{aligned}
$$

where $\varphi_{D}$ is the LISA's Doppler phase, $\left\{u_{+, \times}, w_{+, \times}\right\}$contain an internal summation over all PN orders (see Eq. (5.6) below), $x$ is the PN expansion parameter

$$
x \equiv(M \omega)^{1 / 3},
$$

being $\omega$ the orbital frequency and $\Phi$ is given in Eq. (B5).

In general, $u_{(+, \times), j}$ and $w_{(+, \times), j}$ can be written (at least, up to $2 \mathrm{PN}$ ) as Eq. (5.6). Analyzing those terms, given explicitly in [50], one realizes that all of them have a common factor $\Upsilon(j)$ that only depends on the multipole $j$ we are working with, regardless of the PN order considered

$$
\begin{aligned}
u_{(+, \times), j} & =\sum_{n=0}^{4} x^{n} u_{(+, \times), j}^{(n)}=\Upsilon(j) \sum_{n=0}^{4} x^{n} \hat{u}_{(+, \times), j}^{(n)} \\
& \equiv \Upsilon(j) \hat{u}_{(+, \times), j} \\
w_{(+, \times), j} & =\sum_{n=0}^{4} x^{n} w_{(+, \times), j}^{(n)}=\Upsilon(j) \sum_{n=0}^{4} x^{n} \hat{w}_{(+, \times), j}^{(n)} \\
& \equiv \Upsilon(j) \hat{w}_{(+, \times), j} .
\end{aligned}
$$

This notation is very convenient for computing analytically the derivatives of the waveform. All $(\hat{u}, \hat{w})_{(+, \times), j}^{(n)}$ can be found in Appendix B 2. The factors $Y(j)$, for each multipole, are

$$
\begin{array}{ll}
\Upsilon(j=1) \equiv s \frac{\delta m}{M} & \Upsilon(j=2) \equiv 1 \\
\Upsilon(j=3) \equiv s \frac{\delta m}{M} & \Upsilon(j=4) \equiv s^{2} \\
\Upsilon(j=5) \equiv s^{3} \frac{\delta m}{M} & \Upsilon(j=6) \equiv s^{4},
\end{array}
$$

where $s \equiv \sin \iota=|\hat{\mathbf{L}} \times \hat{\mathbf{N}}|$ and $c \equiv \cos \iota=-\hat{\mathbf{L}} \cdot \hat{\mathbf{N}}$. The source location in the sky $\hat{\mathbf{N}}$ and the orbital angular momentum $\hat{\mathbf{L}}$ can be described by the polar angles $\left(\theta_{N}, \phi_{N}\right)$ and $\left(\theta_{L}, \phi_{L}\right)$ with respect to the solar system barycentre frame.

With all these considerations, the gravitational waveform, given by Eq. (5.4), can be rewritten as follows

$$
h_{j}^{(i)}(t)=\frac{\sqrt{3}}{2} 2 M \eta \frac{1}{D_{L}} x^{2} A_{j} \cos \left(\frac{j}{2} \Phi+\varphi_{p, j}+\varphi_{D}\right),
$$

where 


$$
\begin{gathered}
A_{j}=|\Upsilon(j)|\left[\left(\hat{u}_{+, j} F_{+}+\hat{u}_{\times, j} F_{\times}\right)^{2}\right. \\
\left.+\left(\hat{w}_{\times, j} F_{\times}+\hat{w}_{+, j} F_{+}\right)^{2}\right]^{1 / 2}, \\
\varphi_{p, j}=\tan ^{-1}\left[\frac{-\left(\hat{w}_{\times, j} F_{\times}+\hat{w}_{+, j} F_{+}\right)}{\left(\hat{u}_{+, j} F_{+}+\hat{u}_{\times, j} F_{\times}\right)}\right] .
\end{gathered}
$$

Note that $Y(j)$ cancels in $\varphi_{p, j}$ expression, which prevents a lot of divergence problems in the numerical computation of the Fisher matrix. See Appendix B 1 for further details regarding the waveform in the frequency domain.

\section{RESULTS}

The signal model considered here depends on 11 independent parameters, and it is rather natural to consider the choice

$$
\lambda=\left\{\cos \theta_{N}, \phi_{N}, \cos \theta_{L}, \phi_{L}, \ln D_{L}, t_{c}, \phi_{c}, \beta, \sigma, \ln \mathcal{M}, \ln \mu\right\}
$$

that we adopt in this study, in order for easy comparison with previous existing results. $t_{c}$ and $\phi_{t}$ are the time and phase at coalescence, and $\beta$ and $\sigma$ are the so-called spinorbit and spin-spin parameters. We usually set $t_{c}=\phi_{c}=$ $\beta=\sigma=0$ in our analysis.

The code we use to compute the Fisher matrix is an extension of a previous one developed by Vecchio [61]. Our code allows for multiple choices: selection of PN order in amplitude and phase, number of harmonics, and also different sets of independent parameters. In particular, for the FWF it is more convenient to consider a different choice of the mass parameters to avoid divergences. Therefore, sometimes we consider the mass parameter combination $\{\delta m, M\}$, where $\delta m=m_{1}-m_{2}$ instead of $\{\mathcal{M}, \mu\}$, although we always express the results for comparison with respect to the later choice. The relation of the variance-covariance matrix components, with respect to the different parameters, are

$$
\begin{aligned}
\Sigma^{\mathcal{M M}}= & \left(\frac{\partial \mathcal{M}}{\partial \delta m}\right)^{2} \Sigma^{\delta m \delta m}+\left(\frac{\partial \mathcal{M}}{\partial M}\right)^{2} \Sigma^{M M} \\
& +2 \frac{\partial \mathcal{M}}{\partial M} \frac{\partial \mathcal{M}}{\partial \delta m} \Sigma^{M \delta m}, \\
\Sigma^{\mu \mu}= & \left(\frac{\partial \mu}{\partial \delta m}\right)^{2} \Sigma^{\delta m \delta m}+\left(\frac{\partial \mu}{\partial M}\right)^{2} \Sigma^{M M} \\
& +2 \frac{\partial \mu}{\partial M} \frac{\partial \mu}{\partial \delta m} \Sigma^{M \delta m}, \\
\Sigma_{\mathcal{M} \mu}=\frac{\partial \mathcal{M}}{\partial \delta m} & \frac{\partial \mu}{\partial \delta m} \Sigma^{\delta m \delta m}+\frac{\partial \mathcal{M}}{\partial M} \frac{\partial \mu}{\partial M} \Sigma^{M M} \\
+ & \left(\frac{\partial \mathcal{M}}{\partial M} \frac{\partial \mu}{\partial \delta m}+\frac{\partial \mathcal{M}}{\partial \delta m} \frac{\partial \mu}{\partial M}\right) \Sigma^{M \delta m},
\end{aligned}
$$

where

$$
\begin{gathered}
\frac{\partial \mathcal{M}}{\partial M}=\frac{\mathcal{M}}{20 \mu}\left[5+\frac{\delta m^{2}}{M^{2}}\right], \\
\frac{\partial \mathcal{M}}{\partial \delta m}=-\frac{3 \mathcal{M}}{10 \mu} \frac{\delta m}{M}, \\
\frac{\partial \mu}{\partial M}=\frac{1}{4}+\frac{\delta m^{2}}{4 M^{2}} \\
\frac{\partial \mu}{\partial \delta m}=-\frac{\delta m}{2 M} .
\end{gathered}
$$

Unfortunately, for the equal mass case, the Jacobian of the transformation between $\{\delta m, M\}$ and $\{\mathcal{M}, \mu\}$ is singular, and also the Fisher matrix presents a coordinate singularity depending on the choice of mass parameters and the waveform model used. Since we are still interested in the errors in $\ln \mathcal{M}$ and $\ln \mu$ we convert them using the same previous formulas. For the unequal masses, we find that computing the errors in $\{\delta m, M\}$ and then converting gives the same result as simply computing the errors in $\{\mathcal{M}, \mu\}$ directly. Further details are discussed in Appendix A.

We have checked that our results agree with Vecchio's code for the RWF, and with Sintes and Vecchio [24] at the $0.5 \mathrm{PN}-2 \mathrm{PN}$ order in amplitude and phase, respectively. We use numerical integration and matrix inversion routines from the gs 1 library that, for some particular cases, we have checked against Mathematica.

We work with the full $11 \times 11$ Fisher matrix for both RWF and FWF. There are parameter configurations for which either the numerical integrations or the inversion of such a large matrix tend to fail (or the results do not have

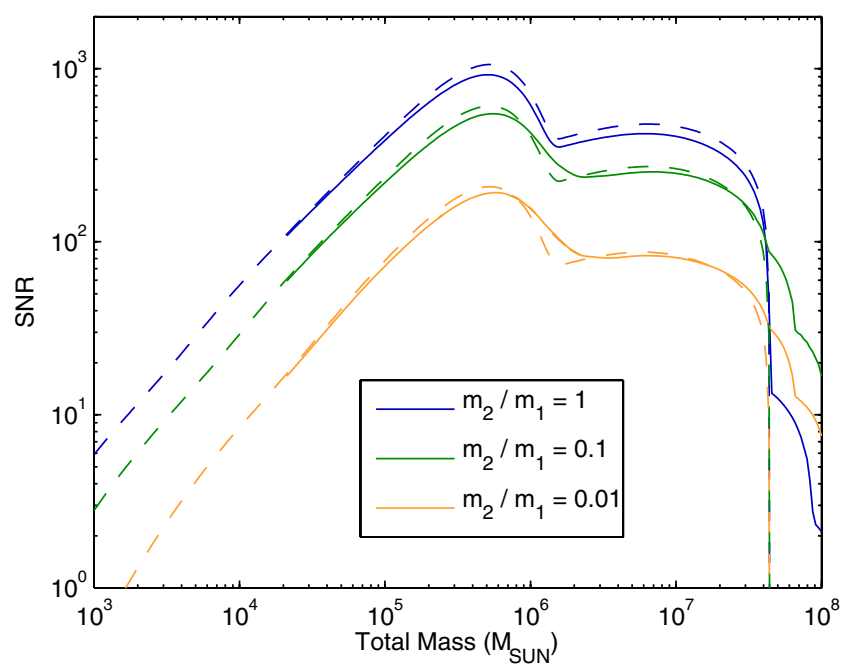

FIG. 1 (color online). SNR versus total mass for the mass ratios of $1,0.1$, and 0.01 for an integration time of 1 year. The solid lines correspond the FWF and the dashed lines to RWF. The sources are at redshift $z=1$, corresponding to a luminosity distance of $D_{L}=6.64 \mathrm{Gpc}$, with fixed angles given by $\cos \theta_{N}=$ $-0.6, \phi_{N}=1, \cos \theta_{L}=0.2$, and $\phi_{L}=3$. 

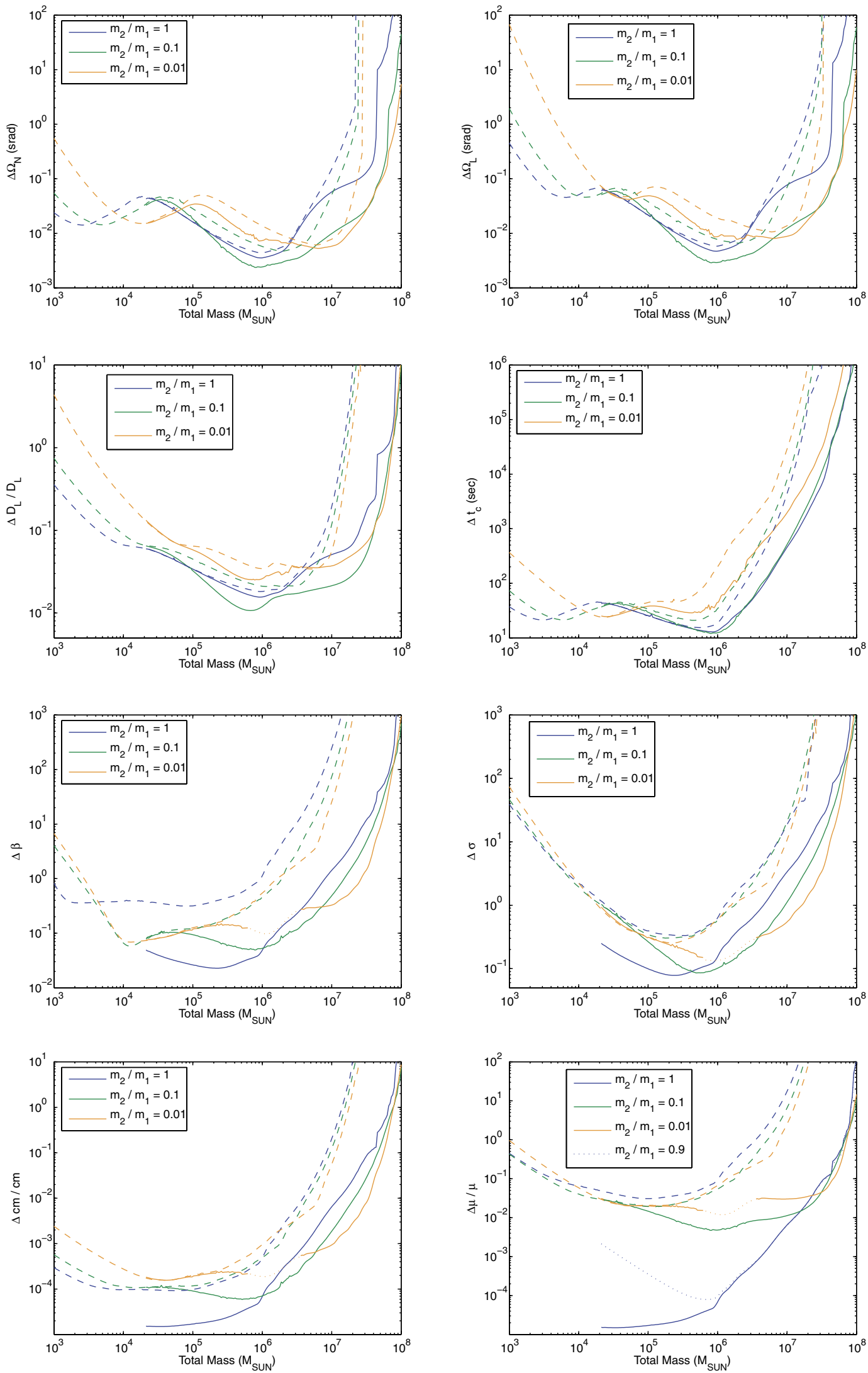

FIG. 2 (color online). Errors versus total mass for the same case as Fig. 1. The solid lines correspond to FWF and the dashed lines to RWF. 
the desired accuracy). The reason is that the Fisher-matrix is often ill conditioned. Because of that, we have limited our study up to a mass ratio $m_{2} / m_{1}=0.01$.

\section{A. Impact of the FWF: General trends}

Given the extensive parameter space to be explored, we start by considering sources with a fixed location and orientation given by $\cos \theta_{N}=-0.6, \phi_{N}=1, \cos \theta_{L}=$ 0.2 , and $\phi_{L}=3$. This is the same choice as in [26], and, as we will point out later, it corresponds to a case for which the SNR and the mass errors are similar to the typical average ones. Note that best and worst cases can span several orders of magnitude. For this set of angles, we consider sources at redshift $z=1$, corresponding to a luminosity distance of $D_{L}=6.64 \mathrm{Gpc}$ and we study the effects on the SNR and parameter estimation using the RWF and the FWF as function of the binary total mass.

In Fig. 1 we plot the SNR computed using the RWF and the FWF as a function of the total mass of the binary system. For systems whose total mass $M<4 \times 10^{7} M_{\odot}$ the RWF overestimates in general the SNR by a few percent. This was already pointed out in [26]. It is also a known fact that the Newtonian amplitude is about $7 \%$ higher than the 2.5PN order amplitude and also than the amplitude obtained by numerical simulations over the last few orbits before merger. For binaries with $M>4 \times$ $10^{7} M_{\odot}$ the second harmonic is no longer visible in the LISA band and higher harmonics, and therefore the use of FWF, play an important role extending the mass reach for supermassive black holes. The "jumps" at high masses are due to the low-frequency cutoff to the instrument at $5 \times$ $10^{-5} \mathrm{~Hz}$. In the case of the FWF we have limited our study to systems with $M>2 \times 10^{4} M_{\odot}$ due to our limited computational resources.

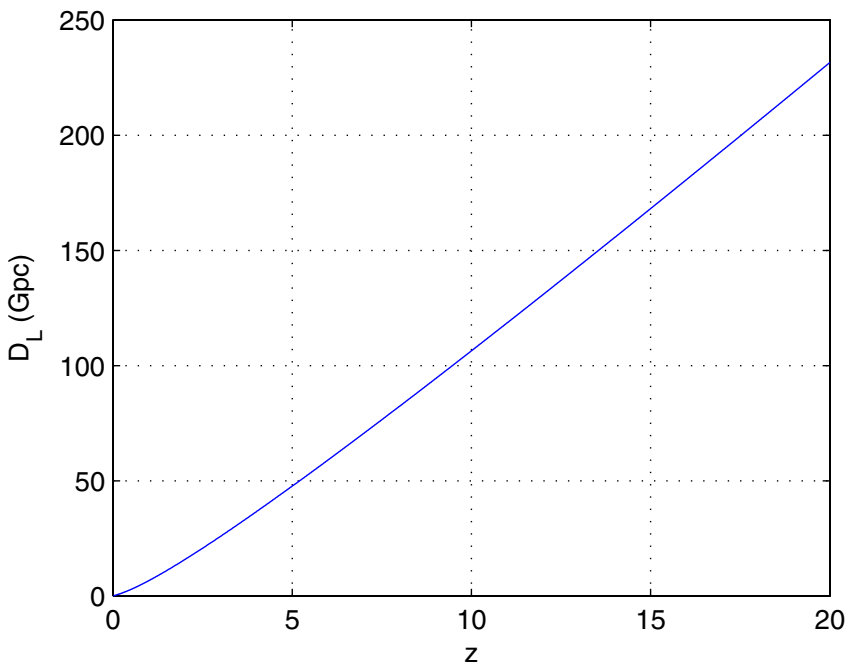

FIG. 3 (color online). The luminosity distance as a function of redshift for a flat universe described by the cosmological parameters: $H_{0}=71 \mathrm{~km} \mathrm{~s}^{-1} \mathrm{Mpc}^{-1}, \Omega_{m}=0.27$, and $\Omega_{\Lambda}=0.73$.
For the same configuration, in Fig. 2 we represent the errors of the most relevant parameters as function of the total mass for different mass ratios. The Fisher matrix has been evaluated assuming the black hole spins to be zero, so that the spin-orbit and spin-spin parameters, $\beta$ and $\sigma$, respectively, are equal to zero. In all cases, the errors are smaller for the FWF. For a total mass $M<10^{5} M_{\odot}$ the improvements are modest, except for the mass estimation for nearly equal masses, for which the errors in $\mathcal{M}$ and $\mu$ are of the same order; while these improvements are considerable for $M>5 \times 10^{6} M_{\odot}$. This betterment is not due to an increase of SNR, but to the higher harmonics that contribute to disentangle the source parameters. For the equal mass case, the errors in $\mu$ improve up to 3 orders of magnitude at $M=10^{5} M_{\odot}$. In general the measurements of the masses improves by more than an order of magnitude for $M>10^{6} M_{\odot}$. We find interesting to add the case $m_{2} / m_{1}=0.9$ obtained by the FWF (dotted dark line, in the lower right panel) for comparison. Using the FWF the luminosity distance can be measured to better than $10 \%$ up to $3-4 \times 10^{7} M_{\odot}$ for this particular source location, while using RWF the errors are several orders of magnitude larger for these large masses. Similarly, the error box in the sky improves significantly at $10^{7} M_{\odot}$, although this sky position, as we will see below, was not one of the most favorable ones.

Using the FWF and a mass ratio of $m_{2} / m_{1}=0.01$, there were several cases in which the errors we obtained did not have the desired accuracy. Those cases have been marked in Fig. 2 by replacing the solid line by a dotted line.

LISA will also be able to measure gravitational waves from massive black hole coalescences to large redshift, making possible to study the merger history of black holes.

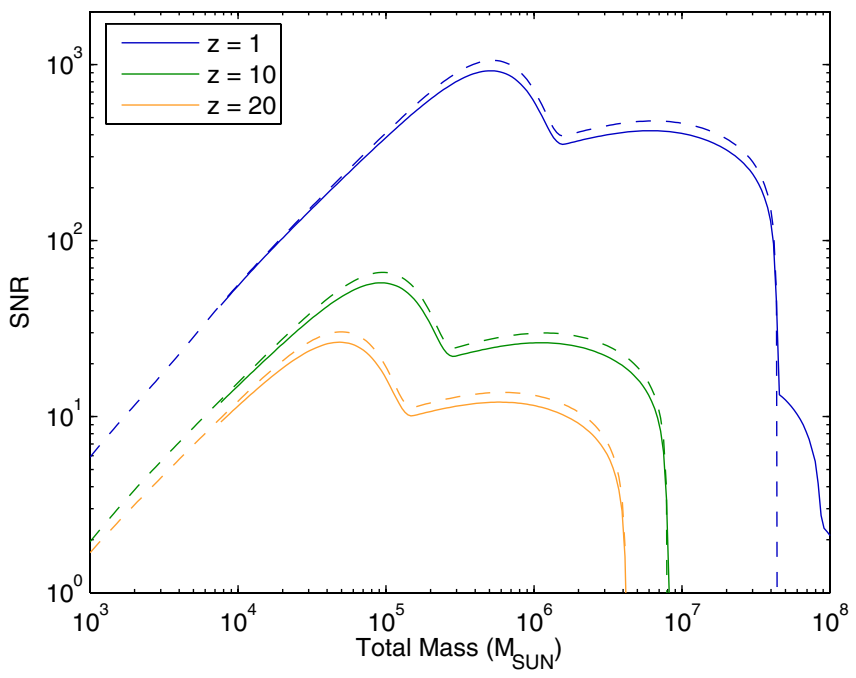

FIG. 4 (color online). SNR versus total mass for various values of the source redshift. We consider equal mass binary systems with fixed angles given by $\cos \theta_{N}=-0.6, \phi_{N}=1, \cos \theta_{L}=$ 0.2 , and $\phi_{L}=3$. The solid lines correspond the FWF and the dashed lines to RWF. 


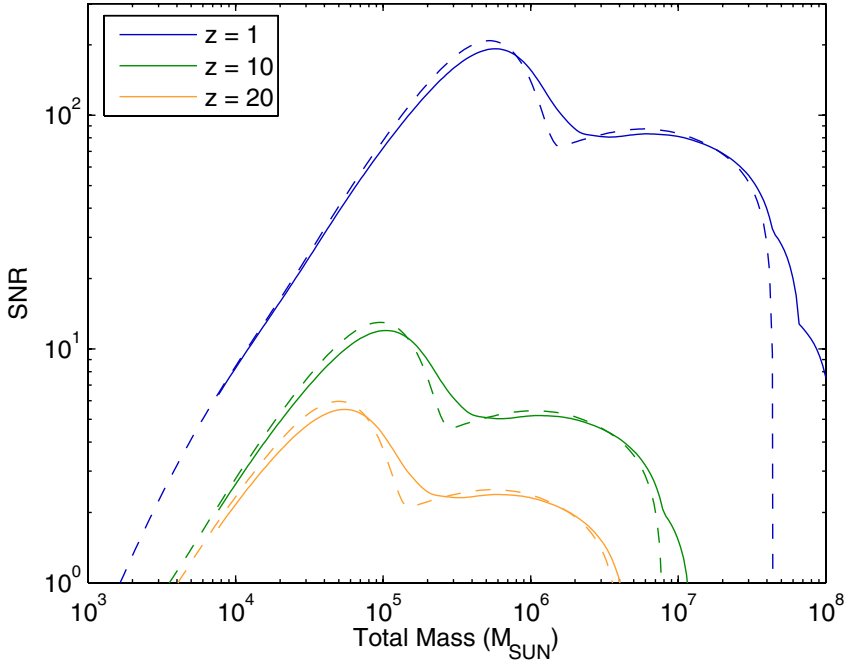

FIG. 5 (color online). The same as Fig. 4 for a mass ratio $m_{2} / m_{1}=0.01$.

Therefore, it is interesting to extend the analysis to higher redshifts. Figure 3 shows the luminosity distance as a function of redshift for a flat universe described by the cosmological parameters: $H_{0}=71 \mathrm{~km} \mathrm{~s}^{-1} \mathrm{Mpc}^{-1}, \Omega_{m}=$ 0.27 , and $\Omega_{\Lambda}=0.73$. In Figs. 4 and 5 we plot the SNR versus total mass for redshifts $z=1,10$, and 20 . The results we obtain are the expected ones since, modulo overall amplitude, the gravitational waves that we measure from a binary with masses $\{\mathcal{M}, \mu\}$ at redshift $z$ are those of a local system with masses $\{(1+z) \mathcal{M},(1+z) \mu\}$. The SNR decreases with $z$, not only due to the distance, but also because the frequency of the signal is redshifted and the total effective noise for LISA is higher at lower frequencies.

In Fig. 6 we plot the distance measurement error, angular resolution, and mass measurement errors for LISA observations of the final year of supermassive black hole inspirals. The fiducial sources are at $z=1,10$, and 20. The waveform considered are the FWF at $2 \mathrm{PN}$ order and the RWF with $\cos \theta_{N}=-0.6, \phi_{N}=1, \cos \theta_{L}=0.2, \phi_{L}=3$, and $\beta=\sigma=0$. For simplicity we display the curves corresponding to the equal mass case, but, as expected, other mass ratios follow the same trend. All curves drift with the redshift, but qualitatively the impact of FWF versus RWF is the same as for $z=1$, but for different mass ranges.

\section{B. Exploring the parameter space}

As we mentioned in the previous section, the error measurements are very sensitive - they vary by orders of magnitude - to the true value of the source parameters; in order to give meaningful results, one therefore is forced to explore a large parameter space. We do this (i) by considering sources on an isotropic grid in the sky with a fixed orientation, and (ii) by extensive Monte Carlo simulations for all possible location and orientation of the source with respect to LISA.
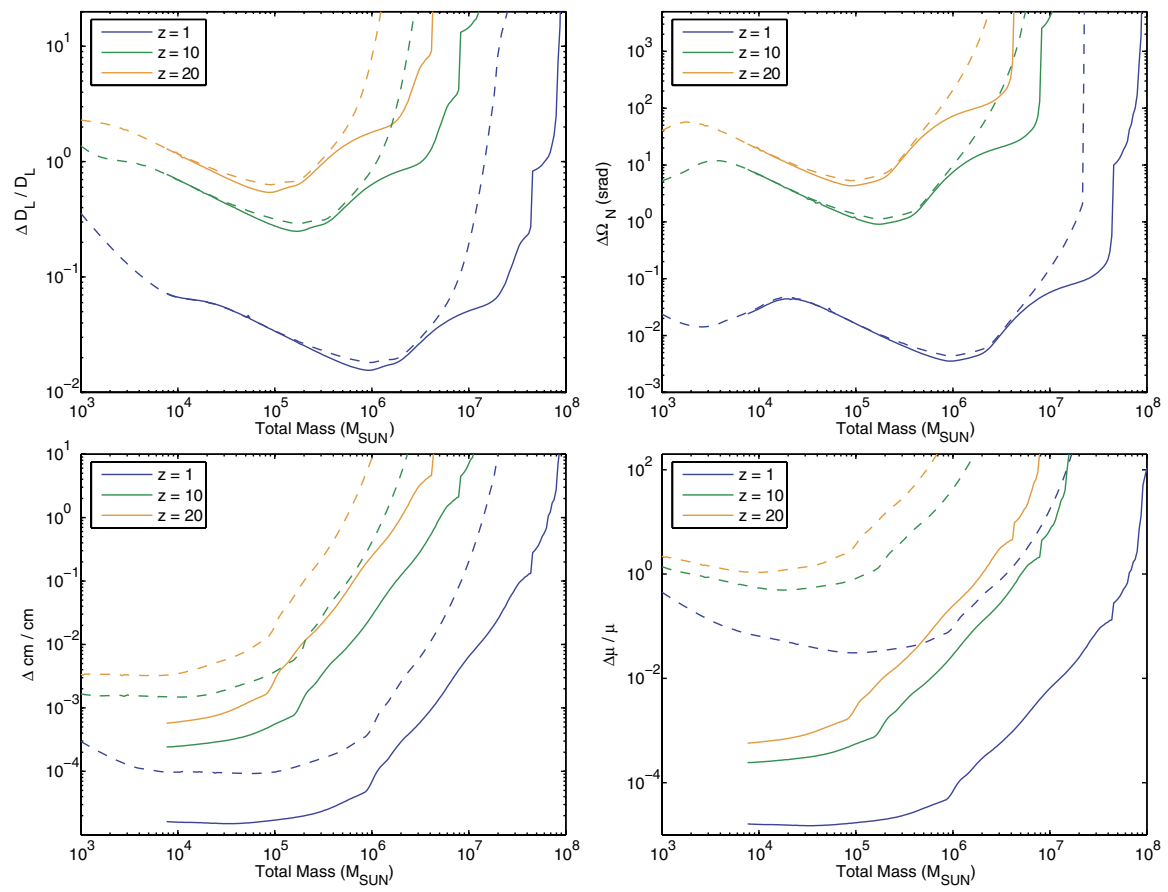

FIG. 6 (color online). Distance measurement error, angular resolution, and mass measurement errors for LISA observations of the final year of equal mass supermassive black hole inspirals. The fiducial sources are at $z=1,10$, and 20 . The waveform considered are the FWF at $2 \mathrm{PN}$ order (solid lines) and the RWF (dashed lines) with $\cos \theta_{N}=-0.6, \phi_{N}=1, \cos \theta_{L}=0.2, \phi_{L}=3$, and $\beta=\sigma=0$. 


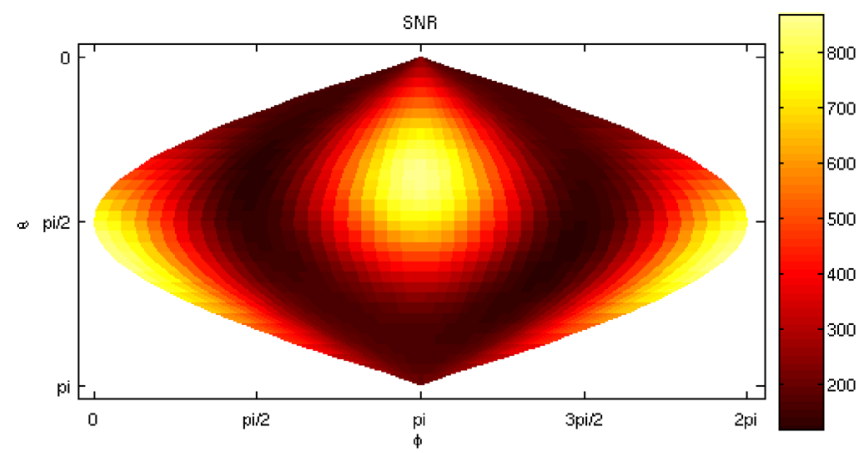

FIG. 7 (color online). Sky map of SNR for LISA observations of the final year of inspirals using FWF. The sources considered correspond to $m_{1}=m_{2}=10^{7} M_{\odot}$, at redshift $z=1$, with orientation angles $\cos \theta_{L}=0.2$ and $\phi_{L}=3$. For all sources we assume the location of LISA at the time of coalescence is $\phi_{\text {LISA }}=0$.

Figure 7 is a sample of a sky map of SNR for LISA observations of the final year of inspirals using FWF. The sources considered correspond to $m_{1}=m_{2}=10^{7} M_{\odot}$ at redshift $z=1$, with orientation angles $\cos \theta_{L}=0.2$ and $\phi_{L}=3$. For all sources we assume the location of LISA at the time of coalescence is $\phi_{\text {LISA }}=0$. The SNR over the entire sky covers a range $\sim 100-900$. The SNR is higher for sources located orthogonal to the plane of LISA at the time of coalescence, which corresponds to $\left(\phi_{N}=\right.$ $\left.0^{\circ} ; \theta_{N}=120^{\circ}\right)$ and $\left(\phi_{N}=180^{\circ} ; \theta_{N}=60^{\circ}\right)$, or in general $\left(\phi_{\perp}=\phi_{\mathrm{LISA}} ; \theta_{\perp}=\theta_{\mathrm{LISA}}+30^{\circ}\right)$ and $\left(\phi_{\perp}=\phi_{\mathrm{LISA}}+\right.$ $\left.180^{\circ} ; \theta_{\perp}=\theta_{\text {LISA }}-30^{\circ}\right)$, since the LISA constellation is inclined at an angle of $60^{\circ}$ with respect to the ecliptic. The reason is that most of the SNR is accumulated in the last days before merger. The reader can notice also the nearly symmetric $[(\theta, \phi) \rightarrow(\pi-\theta, \phi+\pi)]$ profiles of Figs. 710 . The small asymmetry shows the relative importance of the Doppler phase modulation.

Figure 8 shows the angular resolution, and the error in the measurements of the luminosity distance and masses over the entire sky for the same case as Fig. 7. To summarize, the parameters of two $10^{7} M_{\odot}$ black holes spiraling toward the final merger at $z=1$ can be measured very accurately depending on the sky location: $\Delta \Omega_{N}$ up to $\sim 10^{-4}$ srad, the luminosity distance to better than $1 \%$, and the masses between $1 \%$ and $10 \%$. Although it is true that for a given source, the higher SNR the better the parameter estimation, e.g., by changing the distance, this cannot be generalized comparing the SNR at different locations in the sky. It is not just a matter of SNR but long observation times that contribute to disentangle and improve the parameter estimation.

In Fig. 9 we show the gain in angular resolution comparing the results obtained using FWF versus RWF for the case $m_{1}=m_{2}=10^{7} M_{\odot}$ as in Fig. 8 . The benefit of using FWF is clear. For all those sky locations in which we obtain the best angular resolution, $\Delta \Omega_{N} \sim 10^{-4}$ srad with the FWF, the corresponding gain is up to 3 orders of magnitude.

Figure 10 represents also the improvement in angular resolution for the unequal mass case $m_{1}=10^{7} M_{\odot}, m_{2}=$ $10^{6} M_{\odot}$. In this case the optimal gain is of $\sim 2$ orders of magnitude, and those correspond to the same sky locations as in the equal mass case analyzed before.

In order to cover completely the full parameter space, we proceed to perform Monte Carlo simulations according to the following: we consider an ensemble of fiducial sources all at redshift $z=1$ (which sets the luminosity
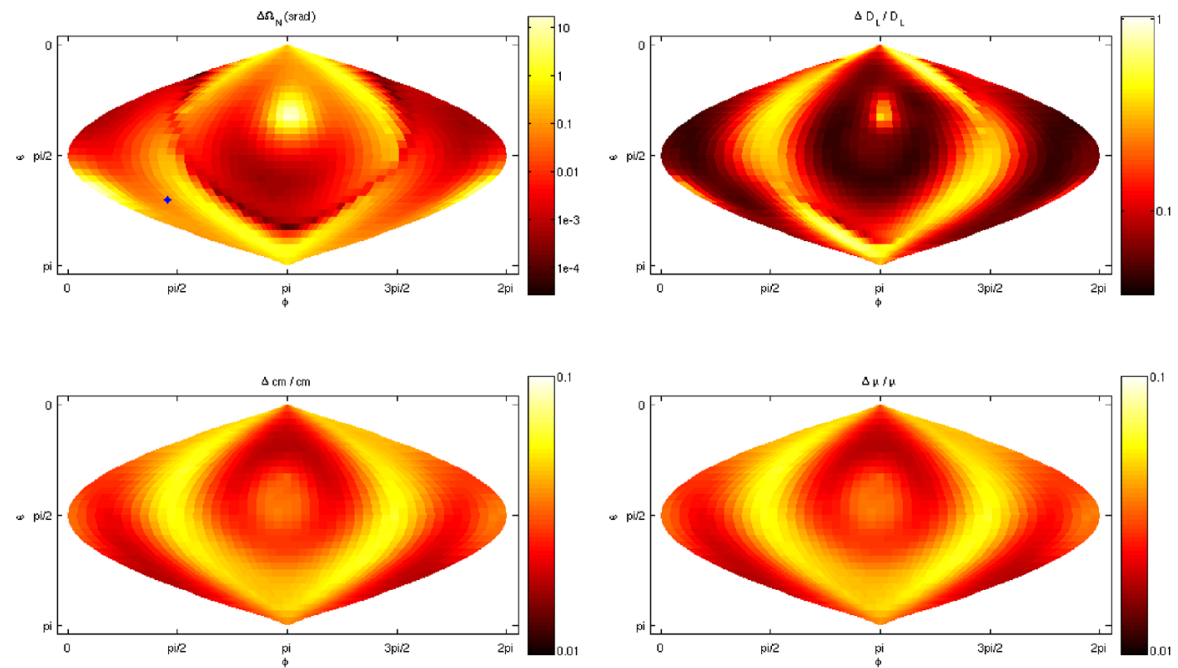

FIG. 8 (color online). Sky maps for the angular resolution, distance measurement error, and mass measurement errors for LISA observations of the final year of inspirals using FWF. As in Fig. 7 these correspond to $m_{1}=m_{2}=10^{7} M_{\odot}$ at redshift $z=1$, with orientation angles $\cos \theta_{L}=0.2, \phi_{L}=3$, and $\beta=\sigma=0$. For all sources we assume the location of LISA at the time of coalescence is $\phi_{\text {LISA }}=0$. The dark blue dot corresponds to $\cos \theta_{N}=-0.6, \phi_{N}=1$, mentioned in the text. 


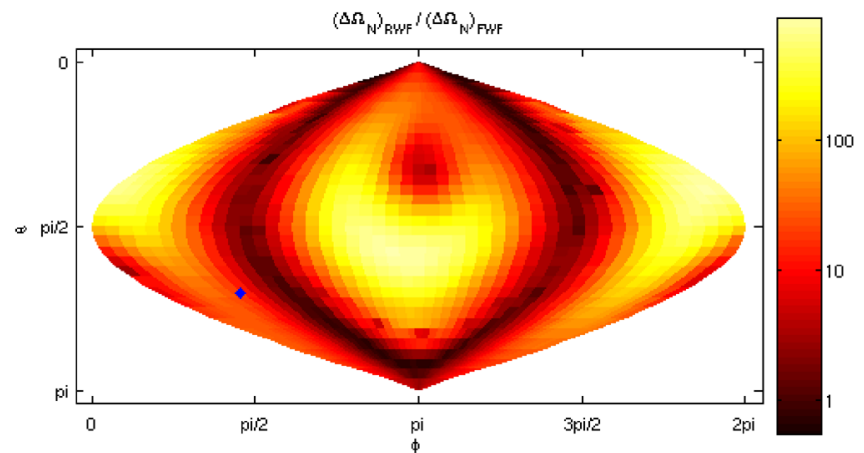

FIG. 9 (color online). Gain in angular resolution comparing the results obtained using FWF versus RWF for the equal mass case $m_{1}=m_{2}=10^{7} M_{\odot}$ with the same assumptions as in Fig. 8 . The dark blue dot corresponds to $\cos \theta_{N}=-0.6, \phi_{N}=1$.

distance $D_{L}=6.64 \mathrm{Gpc}$ ), with zero spins $\beta=\sigma=0$ and we select the value of the masses $m_{1}$ and $m_{2}$. For each set of mass parameters we select randomly the four geometrical angles $\left(\theta_{N}, \phi_{N}, \theta_{L}\right.$, and $\left.\phi_{L}\right)$ from an uniform distribution in $\cos \theta_{N}, \phi_{N}, \cos \theta_{L}$, and $\phi_{L}$, and as far as for the other two parameters, we chose them as $t_{c}=\phi_{c}=0$. The Monte Carlo is done on a 1000 different sets of angles. We present the results in terms of probability distributions.

We have studied six different pairs of masses: (a) $m_{1}=10^{7} M_{\odot} ; m_{2}=10^{7} M_{\odot}$, (b) $m_{1}=10^{7} M_{\odot} ; m_{2}=$ $10^{6} M_{\odot}$, (c) $m_{1}=10^{7} M_{\odot} ; m_{2}=10^{5} M_{\odot}$, (d) $m_{1}=10^{6} M_{\odot}$; $m_{2}=10^{6} M_{\odot}$, (e) $m_{1}=10^{6} M_{\odot} ; \quad m_{2}=10^{5} M_{\odot}$, and (f) $m_{1}=10^{5} M_{\odot} ; m_{2}=10^{5} M_{\odot}$. Figure 11 and Table I summarize the results.

The key result, is that using the FWF the errors are smaller than with the RWF. There are big improvements for the $10^{7} M_{\odot}-10^{7} M_{\odot}$, and $10^{7} M_{\odot}-10^{6} M_{\odot}$ in angular resolution and distance measurement: the angular resolution improves in average 25 and 7.3, respectively; and the

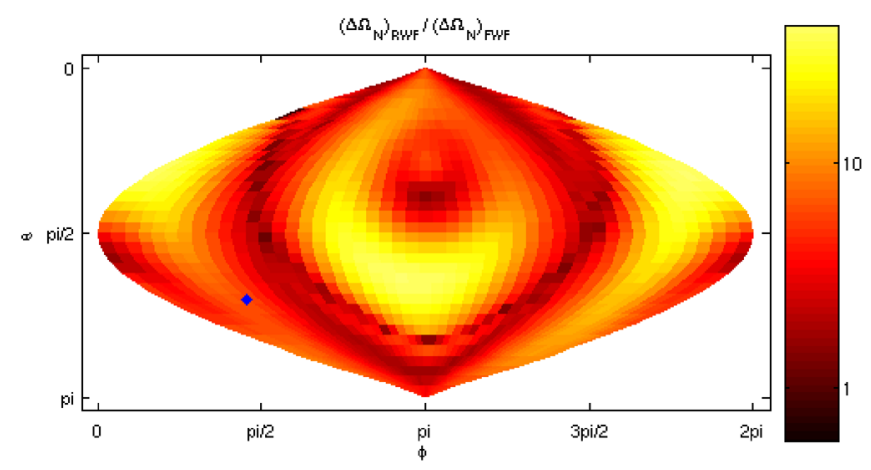

FIG. 10 (color online). Sky map of the gain in angular resolution for LISA observations of the final year of inspirals using FWF versus RWF corresponding to $m_{1}=10^{7} M_{\odot}, m_{2}=10^{6} M_{\odot}$, and $z=1$. We assume all source have the same orientation $\cos \theta_{L}=0.2, \phi_{L}=3$, zero spins $\beta=\sigma=0$ and that LISA is at $\phi_{\text {LISA }}=0$ at the time of coalescence. The dark blue dot corresponds to $\cos \theta_{N}=-0.6, \phi_{N}=1$. luminosity distance by factors of 62 and 6.7, respectively. One should also notice that, in those two cases, those parameters were poorly determined using only the RWF. For the other sets of masses the averaged improvement in angular resolution and luminosity distance are more moderate, between $1.3-3$ for $\Delta \Omega_{N}$, and $1.7-2.7$ for $\Delta D_{L} / D_{L}$. In all cases the masses are determined much more accurate, even by several orders of magnitude in the case of $\mu$, using the FWF. For the equal mass cases, the errors in $\mathcal{M}$ and $\mu$ are of the same order using the FWF.

Because of the different harmonics, the FWF has a much greater richness than RWF that clearly improves the parameter estimation. It is worth mentioning that similar level of improvements were obtained in [24] where the waveform considered was only at the $0.5 \mathrm{PN}-2 \mathrm{PN}$ order in amplitude and phase, respectively, i.e., adding the first and third harmonics. This suggests that the improvement in parameter estimation is mainly due to the inclusion of the third harmonic, which also increases the mass reach of LISA. The importance of the different PN orders has been discussed in detail recently by Arun et al. in [26,27] for some particular cases, and also for ground-based detectors [30].

In Fig. 12 we compare the distribution of the measurement errors for a waveform containing only the second and third harmonics, keeping both amplitude and phase at the 2PN order, with the FWF and the RWF, for the pair of masses $m_{1}=10^{7} M_{\odot}$ and $m_{2}=10^{6} M_{\odot}$. This figure shows how a substantial improvement in parameter estimation is obtained by adding the third harmonic. In this case, the mean and standard deviation of the logarithm of the measurement errors are the following: $\log _{10} \Delta \Omega_{N} / \mathrm{srad}=$ $-2.33 \pm 1.06, \quad \log _{10} \Delta \Omega_{L} / \operatorname{srad}=-2.05 \pm 0.46$, $\log _{10} \Delta D_{L} / D_{L}=-1.22 \pm 0.27, \quad \log _{10} \Delta t_{c} / \mathrm{s}=2.96 \pm$ $0.19, \log _{10} \Delta \mathcal{M} / \mathcal{M}=-2.44 \pm 0.17$, and $\log _{10} \Delta \mu / \mu=$ $-1.57 \pm 0.36$. These results are very close to those obtained for the FWF as can be seen from Table I and are also in agreement with the level of improvement found in [27] when considering only the $0.5 \mathrm{PN}$ order in amplitude.

\section{Premerger localization}

From an astronomical point of view, one of the most attractive features is the possibility that LISA might have enough angular resolution to locate the galaxy or galaxy cluster where the coalescence of a massive black hole takes place and therefore identify potential electromagnetic counterparts. The angular resolution is deduced primarily from the detector's motion around the sun, so one expects that the uncertainty in the angular resolution will not change so much during the last days before merger. Therefore, as discussed in [13], we are interested in analyzing the time dependence of the angular resolution and $\mathrm{SNR}$, as a function of some lookback time $t_{\mathrm{ISCO}}-t_{f}$ prior to coalescence, and measure the importance of the FWF versus RWF. 
(a) $m_{1}=10^{7} M_{\odot} ; m_{2}=10^{7} M_{\odot}$
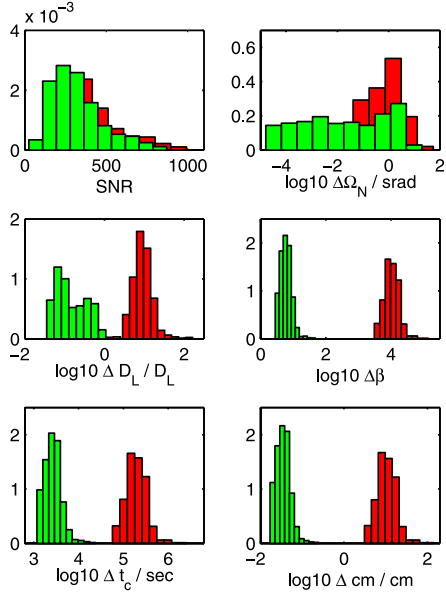

(c) $m_{1}=10^{7} M_{\odot} ; m_{2}=10^{5} M_{\odot}$
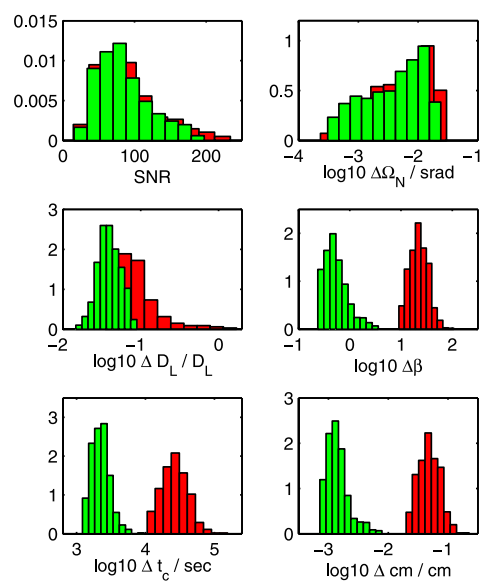

(e) $m_{1}=10^{6} M_{\odot} ; m_{2}=10^{5} M_{\odot}$
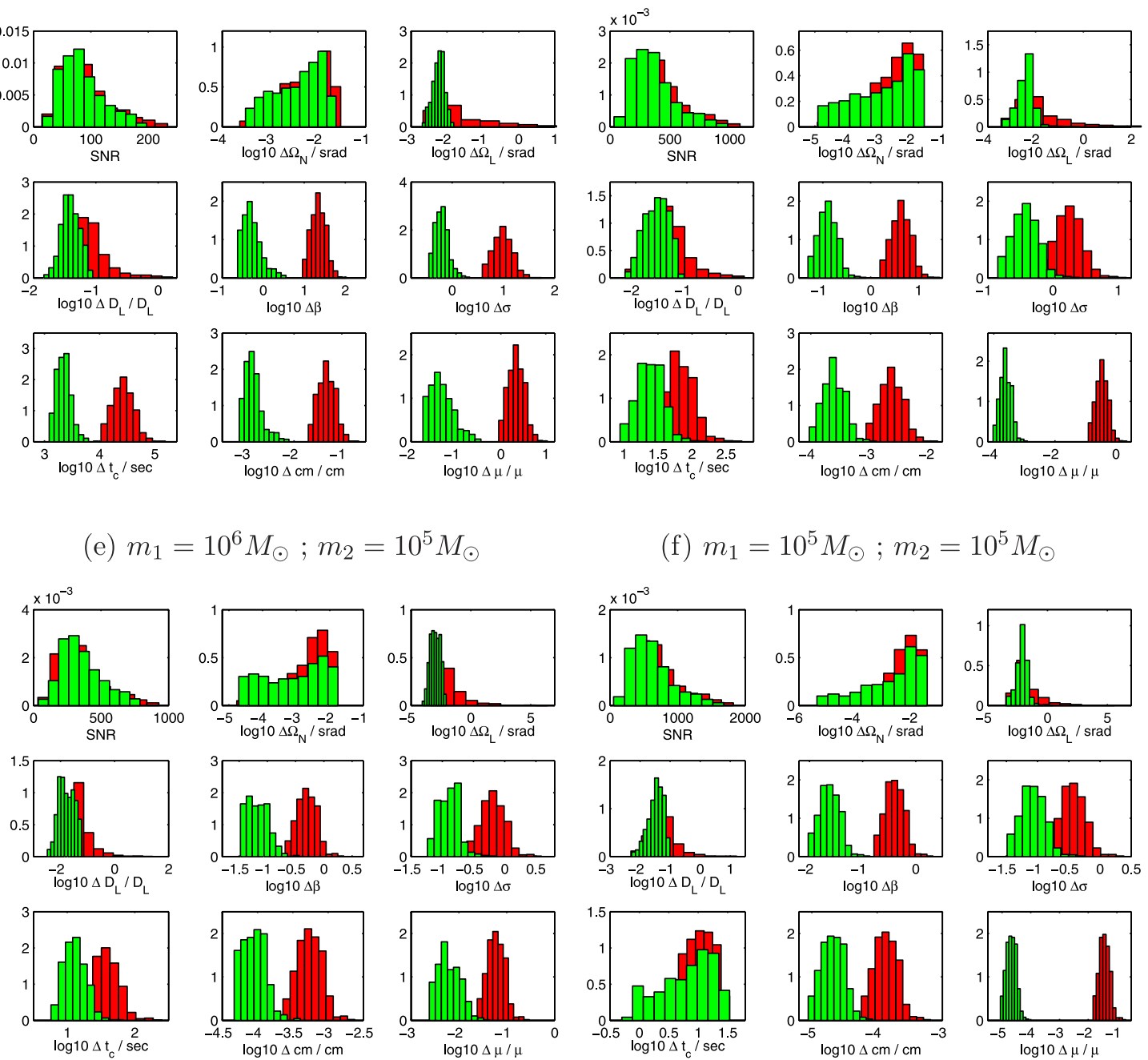

(f) $m_{1}=10^{5} M_{\odot} ; m_{2}=10^{5} M_{\odot}$
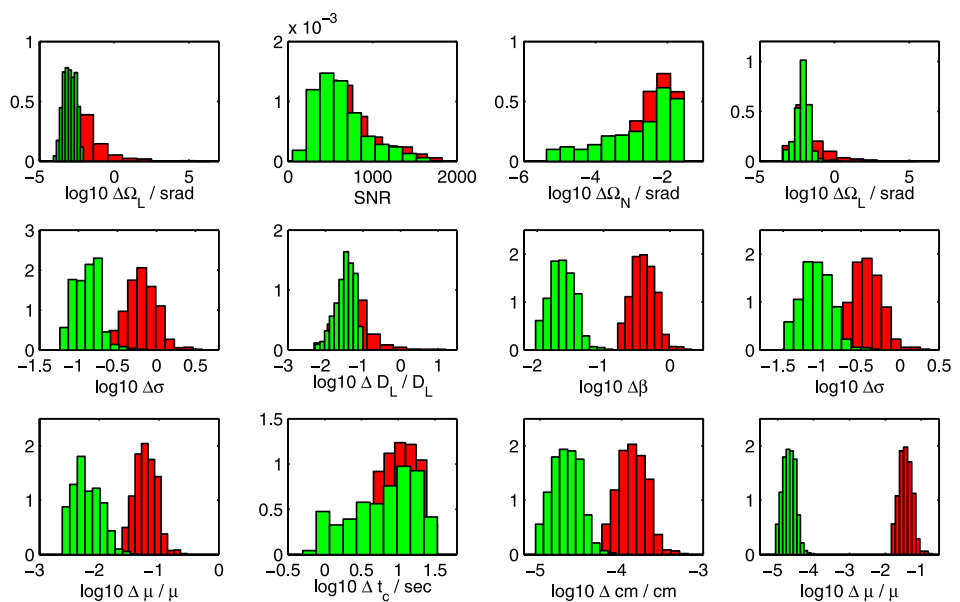

FIG. 11 (color online). The probability distributions of SNR and measurement errors for observations of the final year of supermassive black hole binaries at $z=1$ by LISA. For each pair of masses, the histograms show the result of two Monte Carlo simulations, where 1000 sources have been randomly located and oriented in the sky. The plots compare SNR and the errors for the FWF (front histograms) and the RWF (histograms in the back). 

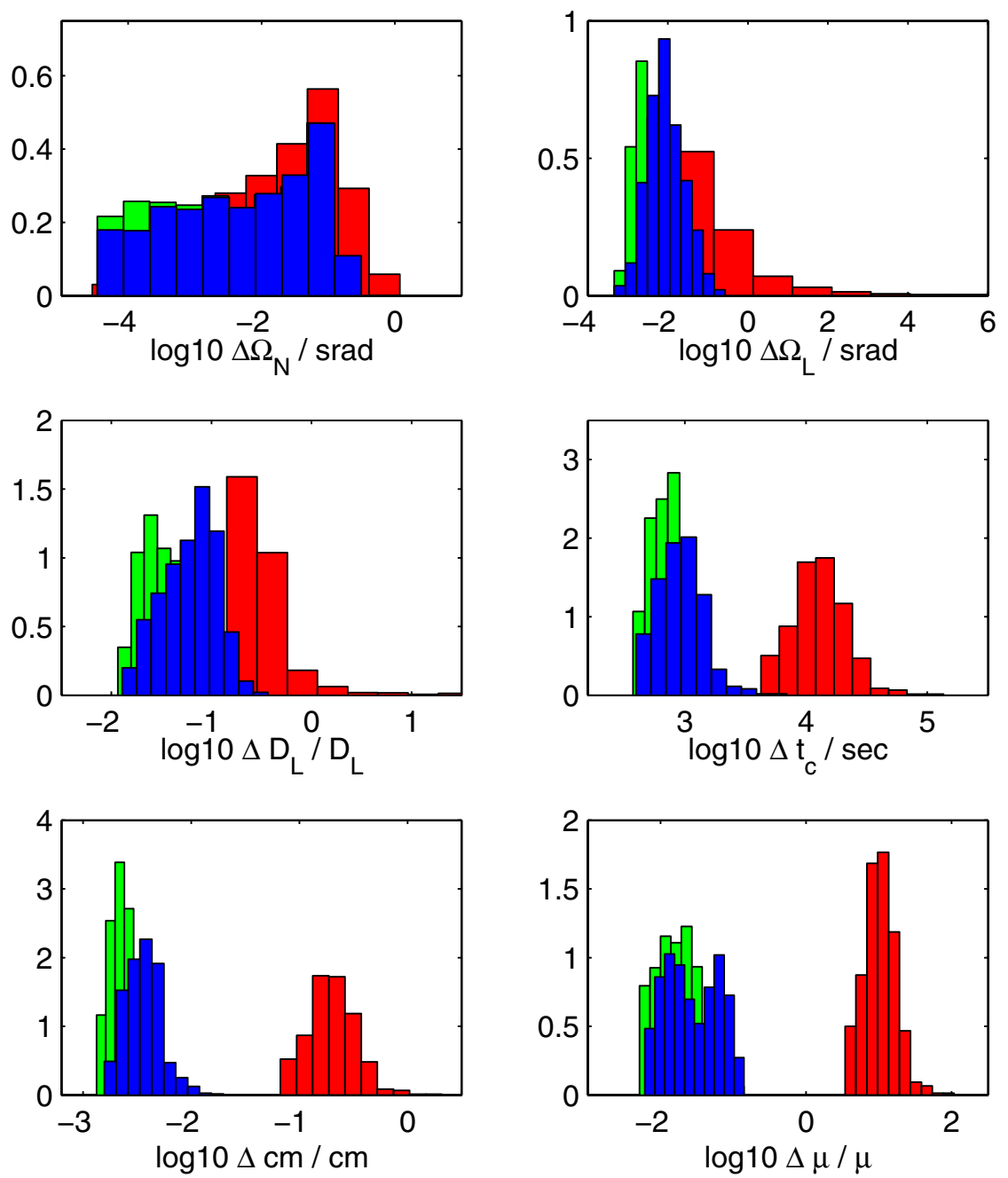

FIG. 12 (color online). Comparison of measurement errors for the FWF [clear gray (green)], the RWF [dark gray (red)] and a waveform containing only the second and third harmonics [black (blue)], corresponding to LISA observations of the final year of inspirals for $m_{1}=10^{7} M_{\odot}, m_{2}=10^{6} M_{\odot}$ and $z=1$.

For fiducial sources at $z=1$ and a given sky location and orientation, Figs. 13 and 14 confirm the importance of the FWF, in particular, for those systems with a higher total mass: $10^{7} M_{\odot}-10^{7} M_{\odot}$ and $10^{7} M_{\odot}-10^{6} M_{\odot}$, while there is not much difference for the $10^{6} M_{\odot}-10^{6} M_{\odot}$ one. For equal masses, only the even multipoles $(2,4,6)$ contribute to the FWF, while for the unequal masses there is contribution from all the six harmonics. The "jumps" in the progressive accumulation of SNR correspond to those times in which a new higher harmonic enters into the LISA band and it is related to the lower-frequency cutoff at $5 \times 10^{-5} \mathrm{~Hz}$ we have imposed. For example, in the $10^{7} M_{\odot}-10^{7} M_{\odot}$ case the contribution of the fourth harmonic becomes relevant around 10 days before coalescence while the second harmonic rapidly increases the SNR 2 days before coalescence. For the unequal mass case $10^{7} M_{\odot}-10^{6} M_{\odot}$ we clearly see the contributions of the second, third, and fourth harmonics.
Using the FWF, not only the uncertainty in sky location decreases but also allows earlier warnings.

\section{SUMMARY AND OUTLOOK}

We have considered LISA observations of supermassive black hole systems in the final stage of inspiral. We have restricted our analysis to systems in circular orbit with negligible spins, modeling the radiation at the full 2PN order, and we have compared with the restricted-2PN. With both waveform models we have determined the meansquare errors associated with the parameter measurements of black hole binaries in the mass range $10^{8} M_{\odot}-10^{5} M_{\odot}$, for equal and unequal mass cases, for a wide range of source locations and orientations.

The conclusions of this work are particularly important with regard to the astrophysical reach of future LISA measurements. Our analysis clearly shows that modeling 
TABLE I. Characterization of the probability distributions of SNR and measurement errors of Fig. 11. For each pair of masses and waveform model used, the mean and standard deviation of the SNR is given, as well as the mean and standard deviation of the logarithm of the measurement errors. The gain factors are computed as $\langle\mathrm{SNR}\rangle_{\mathrm{FWF}} /\langle\mathrm{SNR}\rangle_{\mathrm{RWF}}$ and $10^{\left(\langle x\rangle_{\mathrm{RWF}}-\langle x\rangle_{\mathrm{FWF}}\right)}$, for the SNR and measurement errors, respectively.

\begin{tabular}{|c|c|c|c|c|c|c|}
\hline$\underline{x}$ & $\begin{array}{c}\mathrm{RWF} \\
\langle x\rangle \pm \sigma_{x}\end{array}$ & $\begin{array}{c}\mathrm{FWF} \\
\langle x\rangle \pm \sigma_{x}\end{array}$ & Gain factor & $\begin{array}{c}\mathrm{RWF} \\
\langle x\rangle \pm \sigma_{x}\end{array}$ & $\begin{array}{c}\mathrm{FWF} \\
\langle x\rangle \pm \sigma_{x}\end{array}$ & Gain factor \\
\hline & \multicolumn{3}{|c|}{ (a) $m_{1}=10^{7} M_{\odot} ; m_{2}=10^{7} M_{\odot}$} & \multicolumn{3}{|c|}{ (b) $m_{1}=10^{7} M_{\odot} ; m_{2}=10^{6} M_{\odot}$} \\
\hline SNR & $370 \pm 183$ & $322 \pm 160$ & 0.87 & $258 \pm 127$ & $237 \pm 110$ & 0.92 \\
\hline $\log _{10} \Delta \Omega_{N} / \operatorname{srad}$ & $-0.38 \pm 0.92$ & $-1.78 \pm 1.67$ & 25 & $-1.63 \pm 0.84$ & $-2.50 \pm 1.07$ & 7.3 \\
\hline $\log _{10} \Delta \Omega_{L} / \operatorname{srad}$ & $0.40 \pm 1.20$ & $-1.26 \pm 0.79$ & 46 & $-0.84 \pm 1.20$ & $-2.29 \pm 0.51$ & 28 \\
\hline $\log _{10} \Delta D_{L} / D_{L}$ & $0.96 \pm 0.24$ & $-0.83 \pm 0.39$ & 62 & $-0.56 \pm 0.32$ & $-1.39 \pm 0.27$ & 6.7 \\
\hline $\log _{10} \Delta \beta$ & $4.02 \pm 0.23$ & $0.78 \pm 0.18$ & 1750 & $2.07 \pm 0.22$ & $-0.21 \pm 0.14$ & 190 \\
\hline $\log _{10} \Delta \sigma$ & $2.06 \pm 0.22$ & $1.12 \pm 0.18$ & 8.8 & $1.48 \pm 0.22$ & $0.14 \pm 0.12$ & 22 \\
\hline $\log _{10} \Delta t_{c} / \mathrm{s}$ & $5.28 \pm 0.23$ & $3.42 \pm 0.19$ & 72 & $4.11 \pm 0.22$ & $2.84 \pm 0.14$ & 18 \\
\hline $\log _{10} \Delta \mathcal{M} / \mathcal{M}$ & $1.02 \pm 0.23$ & $-1.46 \pm 0.17$ & 300 & $-0.70 \pm 0.22$ & $-2.64 \pm 0.12$ & 87 \\
\hline \multirow[t]{2}{*}{$\log _{10} \Delta \mu / \mu$} & $2.85 \pm 0.23$ & $-1.46 \pm 0.17$ & 20000 & $1.01 \pm 0.22$ & $-1.77 \pm 0.30$ & 600 \\
\hline & \multicolumn{3}{|c|}{ (c) $m_{1}=10^{7} M_{\odot} ; m_{2}=10^{5} M_{\odot}$} & \multicolumn{3}{|c|}{ (d) $m_{1}=10^{6} M_{\odot} ; m_{2}=10^{6} M_{\odot}$} \\
\hline SNR & $90 \pm 42$ & $85 \pm 36$ & 0.94 & $405 \pm 200$ & $365 \pm 181$ & 0.90 \\
\hline $\log _{10} \Delta \Omega_{N} / \operatorname{srad}$ & $-2.29 \pm 0.48$ & $-2.42 \pm 0.49$ & 1.4 & $-2.58 \pm 0.70$ & $-2.89 \pm 0.93$ & 2.1 \\
\hline $\log _{10} \Delta \Omega_{L} / \operatorname{srad}$ & $-1.50 \pm 1.01$ & $-2.23 \pm 0.16$ & 5.4 & $-1.85 \pm 1.02$ & $-2.40 \pm 0.36$ & 3.5 \\
\hline $\log _{10} \Delta D_{L} / D_{L}$ & $-1.05 \pm 0.27$ & $-1.40 \pm 0.14$ & 2.3 & $-1.34 \pm 0.37$ & $-1.60 \pm 0.23$ & 1.8 \\
\hline $\log _{10} \Delta \beta$ & $1.35 \pm 0.18$ & $-0.27 \pm 0.23$ & 41 & $0.64 \pm 0.20$ & $-0.90 \pm 0.20$ & 34 \\
\hline $\log _{10} \Delta \sigma$ & $0.99 \pm 0.18$ & $-0.20 \pm 0.13$ & 15 & $0.24 \pm 0.20$ & $-0.42 \pm 0.20$ & 4.6 \\
\hline $\log _{10} \Delta t_{c} / \mathrm{s}$ & $4.42 \pm 0.18$ & $3.35 \pm 0.13$ & 12 & $1.84 \pm 0.19$ & $1.39 \pm 0.19$ & 2.8 \\
\hline $\log _{10} \Delta \mathcal{M} / \mathcal{M}$ & $-1.31 \pm 0.18$ & $-2.85 \pm 0.18$ & 34 & $-2.65 \pm 0.19$ & $-3.61 \pm 0.18$ & 9.1 \\
\hline \multirow[t]{2}{*}{$\log _{10} \Delta \mu / \mu$} & $0.34 \pm 0.18$ & $-1.31 \pm 0.27$ & 46 & $-0.46 \pm 0.20$ & $-3.61 \pm 0.18$ & 1420 \\
\hline & \multicolumn{3}{|c|}{ (e) $m_{1}=10^{6} M_{\odot} ; m_{2}=10^{5} M_{\odot}$} & \multicolumn{3}{|c|}{ (f) $m_{1}=10^{5} M_{\odot} ; m_{2}=10^{5} M_{\odot}$} \\
\hline SNR & $348 \pm 170$ & $356 \pm 152$ & 1.0 & $680 \pm 640$ & $620 \pm 310$ & 0.91 \\
\hline $\log _{10} \Delta \Omega_{N} /$ srad & $-2.64 \pm 0.63$ & $-3.10 \pm 0.87$ & 2.9 & $-2.45 \pm 0.69$ & $-2.80 \pm 1.00$ & 2.2 \\
\hline $\log _{10} \Delta \Omega_{L} / \operatorname{srad}$ & $-1.82 \pm 1.22$ & $-2.98 \pm 0.41$ & 15 & $-1.66 \pm 1.21$ & $-2.14 \pm 0.48$ & 3.0 \\
\hline $\log _{10} \Delta D_{L} / D_{L}$ & $-1.35 \pm 0.44$ & $-1.78 \pm 0.29$ & 2.7 & $-1.26 \pm 0.45$ & $-1.48 \pm 0.25$ & 1.7 \\
\hline $\log _{10} \Delta \beta$ & $-0.30 \pm 0.17$ & $-1.17 \pm 0.19$ & 7.4 & $-0.40 \pm 0.18$ & $-1.62 \pm 0.19$ & 17 \\
\hline $\log _{10} \Delta \sigma$ & $-0.19 \pm 0.19$ & $-0.89 \pm 0.16$ & 5.1 & $-0.44 \pm 0.19$ & $-1.07 \pm 0.20$ & 4.4 \\
\hline $\log _{10} \Delta t_{c} / \mathrm{s}$ & $1.57 \pm 0.19$ & $1.11 \pm 0.16$ & 2.9 & $0.97 \pm 0.29$ & $0.80 \pm 0.44$ & 1.5 \\
\hline $\log _{10} \Delta \mathcal{M} / \mathcal{M}$ & $-3.26 \pm 0.17$ & $-4.05 \pm 0.16$ & 6.1 & $-3.86 \pm 0.17$ & $-4.68 \pm 0.17$ & 6.6 \\
\hline $\log _{10} \Delta \mu / \mu$ & $-1.22 \pm 0.18$ & $-2.21 \pm 0.23$ & 9.6 & $-1.44 \pm 0.18$ & $-4.68 \pm 0.17$ & 1760 \\
\hline
\end{tabular}

the inspiral with the full post-Newtonian waveforms, as compared to the restricted-PN ones, not only extends the reach to higher mass systems up to $10^{8} M_{\odot}$, as previously shown in [26], but also improves in general the parameter estimation, and allows for early warnings for systems with a high total mass. There are remarkable improvements in angular resolution and distance measurement for systems with a total mass higher than $5 \times 10^{6} M_{\odot}$, as well as a large improvement in the mass determination. For $\Delta \mu / \mu$, the improvement is more than three orders of magnitude in the case of equal masses.

For binary systems of $10^{7} M_{\odot}-10^{7} M_{\odot}$, and $10^{7} M_{\odot}-$ $10^{6} M_{\odot}$ at redshift $z=1$, the angular resolution improves in average 25 and 7.3, respectively; and the luminosity distance by factors of 62 and 6.7, respectively. Moreover, for the equal mass case $10^{7} M_{\odot}-10^{7} M_{\odot}$, for all those sky locations in which we obtained the best angular resolution, $\Delta \Omega_{N} \sim 10^{-4}$ srad with the FWF, the gain in $\Delta \Omega_{N}$ is up to
3 orders of magnitude. These results are in agreement with those recently found in [27].

These improvements are related to the fact that the FWF has a much greater richness than the RWF, due to the presence of the higher harmonics and, in particular, the main contribution to this improvement is associated to the third harmonic.

There are a number of issues that influence these observations: the instrumental lower-frequency cutoff we have imposed and the confusion noise model we have used are two of them. It would also be very interesting to revise these conclusions, by considering black holes with large spins and precession, since it is known that the presence of spins reduces the errors with which the source parameters are measured [19].

Another issue is the fact that the largest improvement happens for systems with a total mass of $5 \times 10^{6} M_{\odot}$ and higher, and although, we are still in a regime of large SNR, 


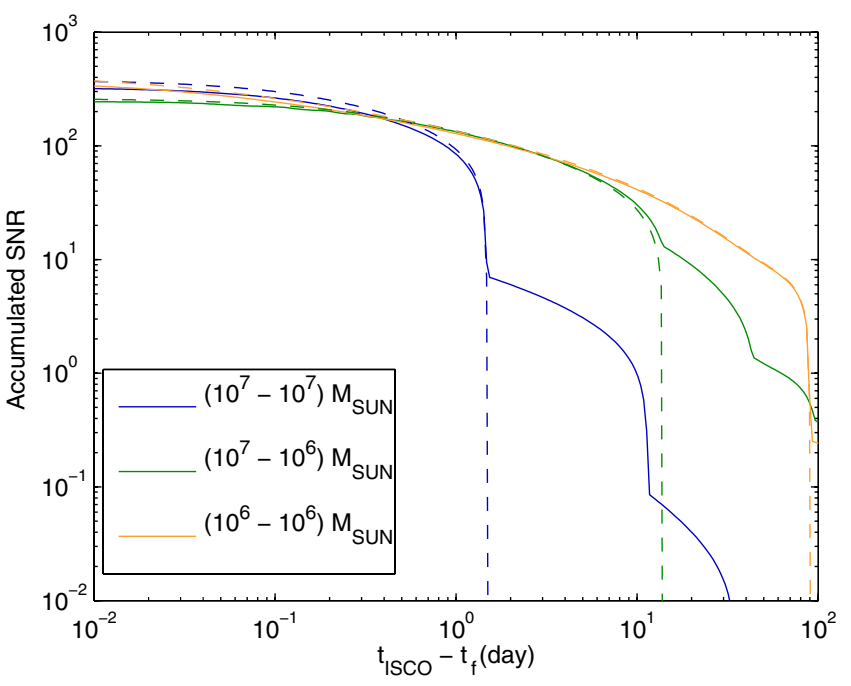

FIG. 13 (color online). The progressive accumulation of SNR as a function of a lookback time; the observations refer to the final year of the inspiral of supermassive black hole as recorded by LISA for fiducial sources at redshift $z=1$, with $\cos \theta_{N}=$ $-0.6, \phi_{N}=1, \cos \theta_{L}=0.2$, and $\phi_{L}=3$. The solid lines correspond the FWF and the dashed lines to RWF.

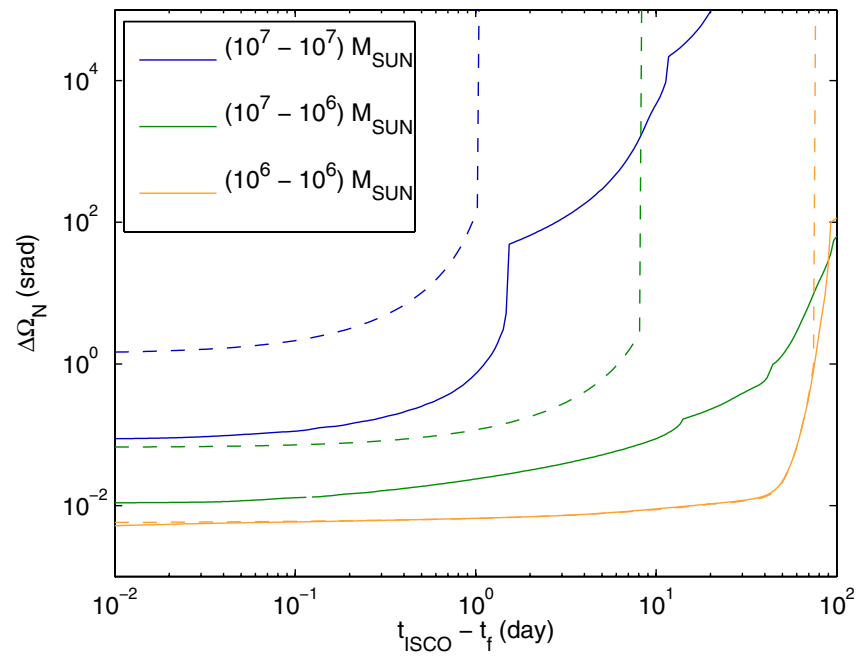

FIG. 14 (color online). Time-dependence angular resolution as a function of a lookback time for the same sources as in Fig. 13. The solid lines correspond the FWF and the dashed lines to RWF.

one could question the validity of the Fisher-matrix approach. Other investigations are currently underway using alternative methods [62].

\section{ACKNOWLEDGMENTS}

We are especially grateful to Alberto Vecchio for encouragement, useful discussions, and for graciously sharing with us his LISA parameter estimation code. We also thank B.S. Sathyaprakash, Bernard Schutz, K. G. Arun,
Stanislav Babak, Chris van den Broeck, Daniel Holz, Scott Hughes, Bala Iyer, and Sascha Husa for many useful discussions. This work was supported by the Spanish Ministerio de Educación y Ciencia research Project No. FPA-2004-03666, and the Conselleria d'Economia Hisenda $\mathrm{i}$ Innovació of the Government of the Balearic Islands. A.M.S. acknowledges the Albert Einstein Institute and the University of Jena for hospitality during several stages of this work.

\section{APPENDIX A: MASS PARAMETERS TRANSFORMATION}

As discussed earlier in Sec. VI it is traditional to parameterize the masses using $\ln \mathcal{M}$ and $\ln \mu$ because of their appearance in the waveform phase. However the higher
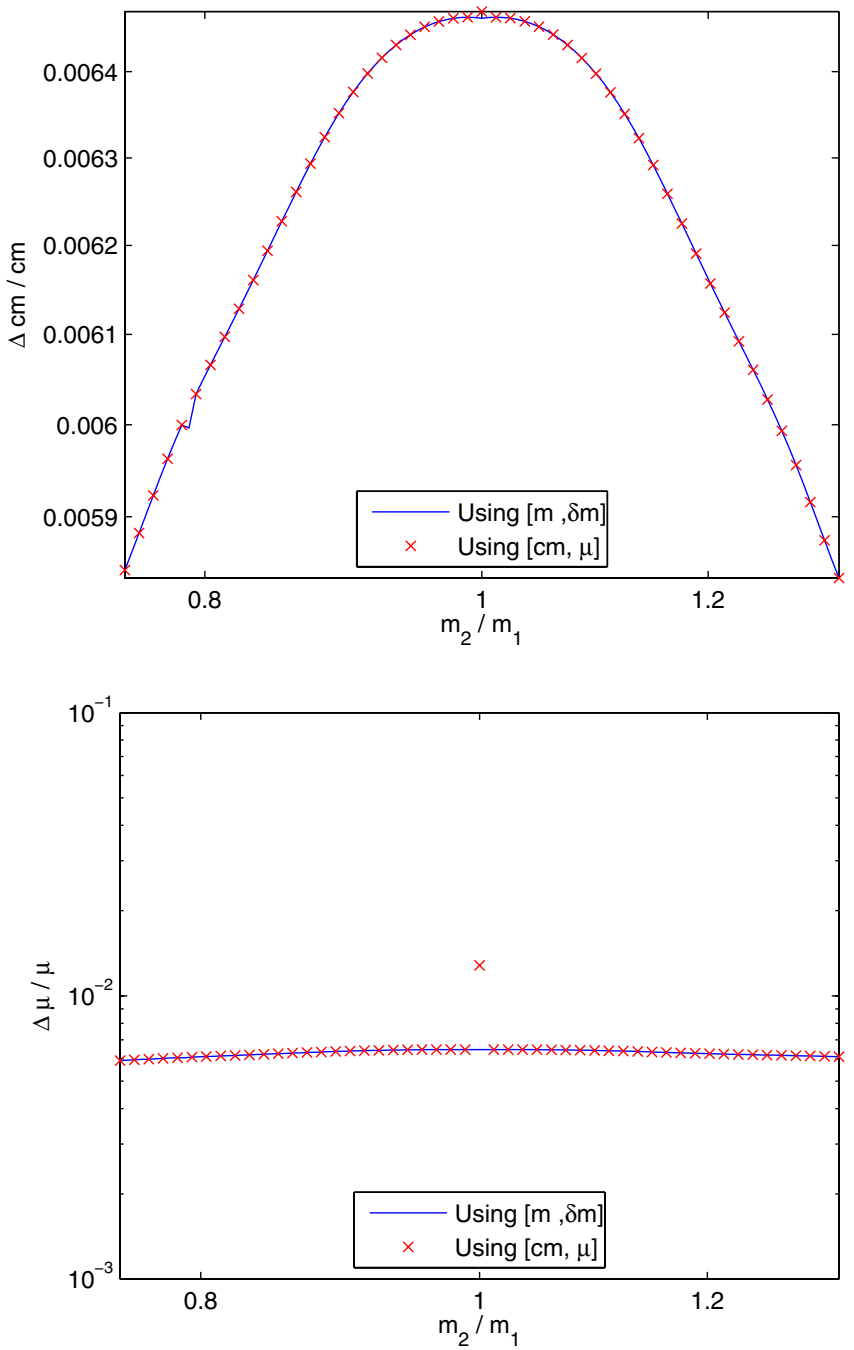

FIG. 15 (color online). Mass measurements using the FWF for binary systems with a total mass $M=10^{7} M_{\odot}$. The solid lines correspond to compute the errors in $\{\delta m, M\}$ and converting them, by mean of Eqs. (6.2), (6.3), (6.4), (6.5), (6.6), (6.7), and (6.8), into $\{\mathcal{M}, \mu\}$, and crosses correspond to compute the errors in $\{\mathcal{M}, \mu\}$ directly. 
order PN amplitude terms depend on the mass difference $\delta m$ and total mass $M$. It is a matter of choice to work with one or another set of parameters. Errors in $\{\mathcal{M}, \mu\}$ can be computed easily, in principle, given the errors in $\{\delta m, M\}$ by mean of Eqs. (6.3), (6.4), (6.5), (6.6), (6.7), and (6.8). Unfortunately the Jacobian of the transformation between $\{\mathcal{M}, \mu\}$ and $\{\delta m, M\}$ is singular when $\delta m=0$ leading to problems in evaluating the Fisher matrix.

For unequal masses, we find that computing the errors in $\{\delta m, M\}$ and then converting gives the same result as simply computing the errors in $\{\mathcal{M}, \mu\}$ directly, and this result is independent of the waveforms used FWF or RWF, as it is shown in Figs. 15 and 16. However, depending of our choice of mass parameters and waveform model we use, there appear divergences when evaluating the Fisher matrix for equal masses. In particular, for the RWF we do not trust the $\{\delta m, M\}$ parameterization, and for the FWF we do not trust the $\{\mathcal{M}, \mu\}$. That is, we trust the solid lines
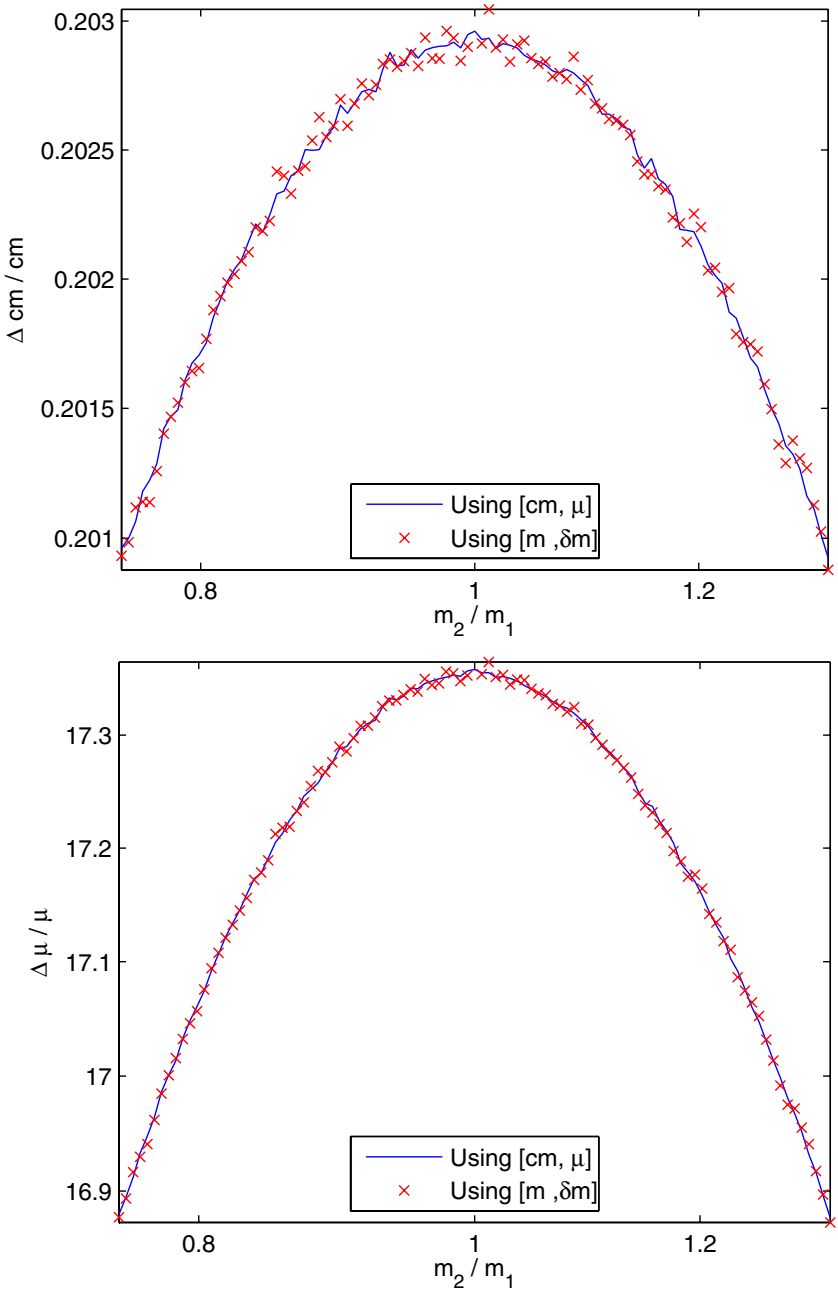

FIG. 16 (color online). Mass measurements using the RWF for binary systems with a total mass $M=10^{7} M_{\odot}$. The solid lines correspond to compute the errors in $\{\mathcal{M}, \mu\}$ directly, and crosses correspond to compute the errors in $\{\delta m, M\}$ first and converting them into $\{\mathcal{M}, \mu\}$.
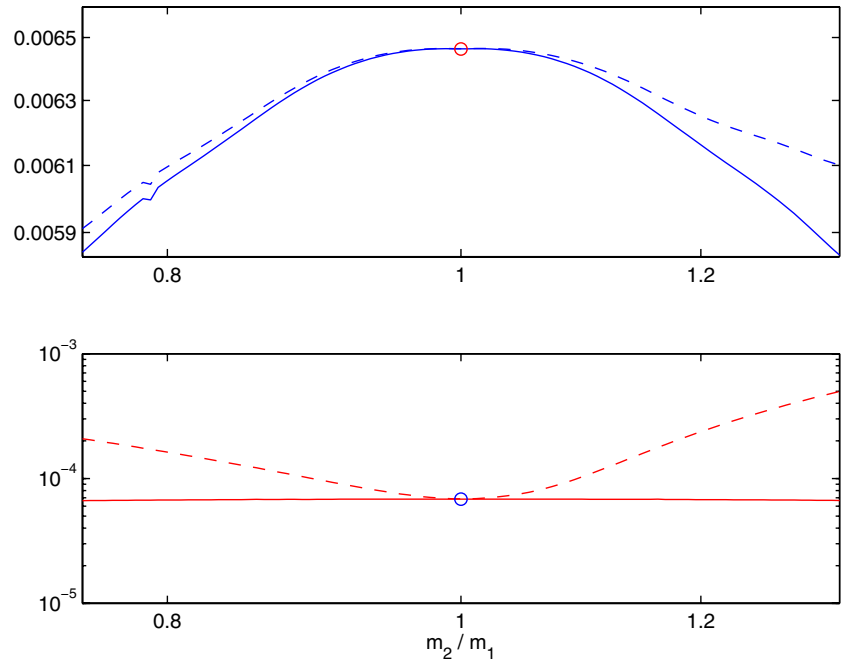

FIG. 17 (color online). Study of the mass parameter measurements in the limit $\delta m \rightarrow 0$ using the FWF with the $\{\delta m, M\}$ mass parameterization for systems with a total mass (top) $M=$ $10^{7} M_{\odot}$ and (bottom) $M=10^{6} M_{\odot}$. In both panels the solid lines correspond to $\Delta \mathcal{M} / \mathcal{M}$, the dashed lines to $\Delta \mu / \mu$ and the circle indicates the equal mass case.

in Figs. 15 and 16, but not the crosses for equal masses, that either do not coincide with the solid lines for the FWF or could not even be computed using the RWF. Because the solid lines did not present any misbehavior nor discontinuities when approaching the equal mass case, we decided to use (independently of the masses) the $\{\mathcal{M}, \mu\}$ parameterization for the RWF and the $\{\delta m, M\}$ for the FWF.

Another aspect we want to study in more detail, is the fact that using the FWF, for equal masses, $\Delta \mathcal{M} / \mathcal{M}$ and $\Delta \mu / \mu$ become identically the same, but this is not true for the RWF, nor for unequal masses. This is a consequence of setting $\delta m=0$ in Eqs. (6.3), (6.4), (6.5), (6.6), (6.7), and (6.8). In Fig. 17 we show that this is not an artifact of our mass transformation due to the Jacobian being singularity at that point but a fact.

\section{APPENDIX B: SOME EXPRESSIONS RELATED WITH THE 2PN INSPIRAL WAVEFORM}

\section{Time, frequency, and phase evolution}

A coalescing binary system evolves by loosing energy and angular momentum $\mathbf{L}$ through emission of gravitational waves of increasing frequency and amplitude. Working with the post-Newtonian approximation, taking into account possible spin motions of each object, $\mathbf{S}_{1}$ and $\mathbf{S}_{2}$, the signal frequency of the second harmonic, $F=$ $2 f_{\text {orb}}$, evolves, up to $2 \mathrm{PN}$ order, according to $[7,19,22,29,30,50]$

$$
\begin{aligned}
\frac{d F}{d t}= & \frac{96}{5} \pi F^{2} \eta x^{5}\left[1-\left(\frac{743}{336}+\frac{11}{4} \eta\right) x^{2}+(4 \pi-\beta) x^{3}\right. \\
& \left.+\left(\frac{34103}{18144}+\frac{13661}{2016} \eta+\frac{59}{18} \eta^{2}+\sigma\right) x^{4}\right],
\end{aligned}
$$


where $x$ is the PN expansion parameter defined in Eq. (5.5), and $\beta$ and $\sigma$ are the so-called spin-orbit and spin-spin parameters, respectively

$$
\begin{gathered}
\beta=\frac{1}{12} \sum_{i=1}^{2}\left[113\left(\frac{m_{i}}{M}\right)^{2}+75 \eta\right]\left(\hat{\mathbf{L}} \cdot \frac{\mathbf{S}_{i}}{m_{i}^{2}}\right), \\
\sigma=\frac{\eta}{48}\left[-247\left(\frac{\mathbf{S}_{1}}{m_{1}^{2}} \cdot \frac{\mathbf{S}_{2}}{m_{2}^{2}}\right)+721\left(\hat{\mathbf{L}} \cdot \frac{\mathbf{S}_{1}}{m_{1}^{2}}\right)\left(\hat{\mathbf{L}} \cdot \frac{\mathbf{S}_{2}}{m_{2}^{2}}\right)\right] .
\end{gathered}
$$

Integrating Eq. (B1), one can derive the time evolution of the gravitational radiation

$$
\begin{aligned}
t(F)= & t_{c}-\frac{5}{256 \eta \pi F} x^{-5}\left[1+\frac{4}{3}\left(\frac{743}{336}+\frac{11}{4} \eta\right) x^{2}\right. \\
& -\frac{8}{5}(4 \pi-\beta) x^{3}+2\left(\frac{3058673}{1016064}+\frac{5429}{1008} \eta\right. \\
& \left.\left.+\frac{617}{144} \eta^{2}-\sigma\right) x^{4}\right]
\end{aligned}
$$

and the phase evolution of the gravitational waveform. For the second harmonic this is

$$
\begin{aligned}
\Phi= & \Phi_{c}-\frac{3}{128 \eta} x^{-5}\left[1+\frac{20}{9}\left(\frac{743}{336}+\frac{11}{4} \eta\right) x^{2}\right. \\
& -(16 \pi-4 \beta) x^{3}+10\left(\frac{3058673}{1016064}+\frac{5429}{1008} \eta\right. \\
& \left.\left.+\frac{617}{144} \eta^{2}-\sigma\right) x^{4}\right] .
\end{aligned}
$$

The gravitational waveform can be computed in the frequency domain using the stationary phase approximation

$$
\tilde{h}(\nu)=\sum_{j=1}^{6}\left[\frac{\tilde{h}_{j}}{2} e^{i\left[\left(2 \pi F t_{c}-\Phi\right) j / 2-\pi / 4-\varphi_{p, j}-\varphi_{D}\right]} \sqrt{\frac{2}{j \frac{d F}{d t}}}\right]_{F=2 \nu / j}
$$

where $\tilde{h}_{j} \equiv \frac{\sqrt{3}}{2} 2 M \eta \frac{1}{D_{L}} x^{2} A_{j}$. Using Eq. (B1), $(d F / d t)^{-1 / 2}$ can be written to $2 \mathrm{PN}$ order as

$$
\frac{1}{\sqrt{\frac{d F}{d t}}}=\sqrt{\frac{5 \pi}{96}} M \eta^{-1 / 2} x^{-11 / 2}\left(\sum_{n=0}^{4} k_{n} x^{n}\right),
$$

where coefficients $k_{n}$ are defined as follows

$$
\begin{aligned}
& k_{0}=1 \\
& k_{1}=0 \\
& k_{2}=\frac{1}{2}\left(\frac{743}{336}+\frac{11}{4} \eta\right) \\
& k_{3}=-\frac{1}{2}(4 \pi-\beta) \\
& k_{4}=\frac{7266251}{8128512}+\frac{18913}{16128} \eta+\frac{1379}{1152} \eta^{2}-\frac{\sigma}{2} .
\end{aligned}
$$

\section{2. $\hat{\boldsymbol{u}}_{(+, \times), j}$ and $\hat{\boldsymbol{w}}_{(+, \times), j}$ up to $2 \mathrm{PN}$}

In Sec. V B we have seen a general form to expand the GW amplitude, of a particular multipole, as a summation of its different PN contributions. The analytical expression of all the terms appearing in Eq. (5.6) can be obtained from [50]. Here we explicitly give all the nonvanishing terms $\hat{u}_{(+, \times), j}^{(n)}$ and $\hat{w}_{(+, \times), j}^{(n)}$ up to $n=4$.

Contributions to $\hat{u}_{+, j}$

$$
\begin{aligned}
& \hat{u}_{+, 1}^{(1)}=-\frac{1}{8}\left(5+c^{2}\right) \\
& \hat{u}_{+, 1}^{(3)}=\frac{1}{192}\left[\left(57+60 c^{2}-c^{4}\right)-2 \eta\left(49-12 c^{2}-c^{4}\right)\right]
\end{aligned}
$$$$
\hat{u}_{+, 1}^{(4)}=-\frac{\pi}{8}\left(5+c^{2}\right)
$$

$\hat{u}_{+, 2}^{(0)}=-\left(1+c^{2}\right)$

$\hat{u}_{+, 2}^{(2)}=\frac{1}{6}\left[\left(19+9 c^{2}-2 c^{4}\right)-\eta\left(19-11 c^{2}-6 c^{4}\right)\right]$

$\hat{u}_{+, 2}^{(3)}=-2 \pi\left(1+c^{2}\right)$

$\hat{u}_{+, 2}^{(4)}=\frac{1}{120}\left[\left(22+396 c^{2}+145 c^{4}-5 c^{6}\right)\right.$

$+\frac{5}{3} \eta\left(706-216 c^{2}-251 c^{4}+15 c^{6}\right)$

$\left.-5 \eta^{2}\left(98-108 c^{2}+7 c^{4}+5 c^{6}\right)\right]$

$$
\hat{u}_{+, 3}^{(1)}=\frac{9}{8}\left(1+c^{2}\right)
$$

$\hat{u}_{+, 3}^{(3)}=-\frac{9}{128}\left[\left(73+40 c^{2}-9 c^{4}\right)-2 \eta\left(25-8 c^{2}-9 c^{4}\right)\right]$

$\hat{u}_{+, 3}^{(4)}=\frac{27}{8} \pi\left(1+c^{2}\right)$

$$
\begin{aligned}
\hat{u}_{+, 4}^{(2)}= & -\frac{4}{3}\left(1+c^{2}\right)(1-3 \eta) \\
\hat{u}_{+, 4}^{(4)}= & \frac{2}{15}\left[\left(59+35 c^{2}-8 c^{4}\right)-\frac{5}{3} \eta\left(131+59 c^{2}-24 c^{4}\right)\right. \\
& \left.+5 \eta^{2}\left(21-3 c^{2}-8 c^{4}\right)\right]
\end{aligned}
$$




$$
\begin{gathered}
\hat{u}_{+, 5}^{(3)}=\frac{625}{384}\left(1+c^{2}\right)(1-2 \eta) \\
\hat{u}_{+, 6}^{(4)}=-\frac{81}{40}\left(1+c^{2}\right)\left(1-5 \eta+5 \eta^{2}\right) .
\end{gathered}
$$

Contributions to $\hat{w}_{+, j}$

$$
\begin{aligned}
& \hat{w}_{+, 1}^{(4)}=\frac{1}{40}\left[11+7 c^{2}+10\left(5+c^{2}\right) \ln 2\right] \\
& \hat{w}_{+, 3}^{(4)}=-\frac{27}{40}\left(1+c^{2}\right)\left[7-10 \ln \frac{3}{2}\right] .
\end{aligned}
$$

\section{Contributions to $\hat{w}_{\times, j}$}

$$
\begin{aligned}
\hat{w}_{\times, 1}^{(1)}= & -\frac{3}{4} c \\
\hat{w}_{\times, 1}^{(3)}= & \frac{c}{96}\left[\left(63-5 c^{2}\right)-2 \eta\left(23-5 c^{2}\right)\right] \\
\hat{w}_{\times, 1}^{(4)}= & -\frac{3 \pi}{4} c \\
\hat{w}_{\times, 2}^{(0)}= & -2 c \\
\hat{w}_{\times, 2}^{(2)}= & \frac{c}{3}\left[\left(17-4 c^{2}\right)-\eta\left(13-12 c^{2}\right)\right] \\
\hat{w}_{\times, 2}^{(3)}= & -4 \pi c \\
\hat{w}_{\times, 2}^{(4)}= & \frac{c}{60}\left[\left(68+226 c^{2}-15 c^{4}\right)\right. \\
& +\frac{5}{3} \eta\left(572-490 c^{2}+45 c^{4}\right) \\
& \left.\quad-5 \eta^{2}\left(56-70 c^{2}+15 c^{4}\right)\right]
\end{aligned}
$$

$$
\begin{aligned}
& \hat{w}_{\times, 3}^{(1)}=\frac{9}{4} c \\
& \hat{w}_{\times, 3}^{(3)}=-\frac{9}{64} c\left[\left(67-15 c^{2}\right)-2 \eta\left(19-15 c^{2}\right)\right] \\
& \hat{w}_{\times, 3}^{(4)}=\frac{27}{4} \pi c
\end{aligned}
$$

$$
\begin{aligned}
& \hat{w}_{\times, 4}^{(2)}=-\frac{8}{3} c(1-3 \eta) \\
& \hat{w}_{\times, 4}^{(4)}= \frac{4}{15} c\left[\left(55-12 c^{2}\right)-\frac{5}{3} \eta\left(119-36 c^{2}\right)\right. \\
&\left.+5 \eta^{2}\left(17-12 c^{2}\right)\right] \\
& \hat{w}_{\times, 5}^{(3)}=\frac{625}{192} c(1-2 \eta) \\
& \hat{w}_{\times, 6}^{(4)}=-\frac{81}{20} c\left(1-5 \eta+5 \eta^{2}\right) .
\end{aligned}
$$

Contributions to $\hat{u}_{\times, j}$

$$
\begin{aligned}
& \hat{u}_{\times, 1}^{(4)}=-\frac{3}{20} c(3+10 \ln 2) \\
& \hat{u}_{\times, 3}^{(4)}=\frac{27}{20} c\left(7-10 \ln \frac{3}{2}\right) .
\end{aligned}
$$

[1] K. Danzmann et al., Max-Planck-Institut für Quantenoptic Report No. MPQ 233, 1998 (unpublished).

[2] K. Danzmann and A. Rüdiger, Classical Quantum Gravity 20, S1 (2003).

[3] http://lisa.jpl.nasa.gov.

[4] O. Dreyer, B. Kelly, B. Krishnan, L. S. Finn, D. Garrison, and R. Lopez-Aleman, Classical Quantum Gravity 21, 787 (2004).

[5] M. C. Miller, Astrophys. J. 618, 426 (2005).

[6] S. A. Hughes and K. Menou, Astrophys. J. 623, 689 (2005).

[7] E. Berti, A. Buonanno, and C. M. Will, Phys. Rev. D 71, 084025 (2005).

[8] K. G. Arun, B. R. Iyer, M.S.S. Qusailah, and B.S. Sathyaprakash, Classical Quantum Gravity 23, L37 (2006).

[9] K. G. Arun, B. R. Iyer, M.S.S. Qusailah, and B.S. Sathyaprakash, Phys. Rev. D 74, 024006 (2006).

[10] B. F. Schutz, Nature (London) 323, 310 (1986).
[11] S. A. Hughes, Mon. Not. R. Astron. Soc. 331, 805 (2002).

[12] D. E. Holz and S. A. Hughes, Astrophys. J. 629, 15 (2005).

[13] B. Kocsis, Z. Haiman, K. Menou, and Z. Frei, Phys. Rev. D 76, 022003 (2007).

[14] J.E. Barnes and L.E. Hernquist, Annu. Rev. Astron. Astrophys. 30, 705 (1992).

[15] K. Menou, Z. Haiman, and V. K. Narayanan, Astrophys. J. 558, 535 (2001).

[16] M. Milosavljevic and E. S. Phinney, Astrophys. J. 622, L93 (2005).

[17] B. Kocsis, Z. Frei, Z. Haiman, and K. Menou, Astrophys. J. 637, 27 (2006).

[18] C. Cutler, Phys. Rev. D 57, 7089 (1998).

[19] A. Vecchio, Phys. Rev. D 70, 042001 (2004).

[20] K. G. Arun, Phys. Rev. D 74, 024025 (2006).

[21] L. Barack and C. Cutler, Phys. Rev. D 69, 082005 (2004).

[22] R. N. Lang and S. A. Hughes, Phys. Rev. D 74, 122001 (2006); 75, 089902(E) (2007).

[23] N. Seto, Phys. Rev. D 66, 122001 (2002). 
[24] A. M. Sintes and A. Vecchio, in Third Amaldi Conference on Gravitational Waves, edited by S. Meshkov, AIP Conf. Proc. (AIP, New York, 2000), p. 403.

[25] T. A. Moore and R. W. Hellings, Phys. Rev. D 65, 062001 (2002).

[26] K. G. Arun, B. R. Iyer, B. S. Sathyaprakash, and S. Sinha, Phys. Rev. D 75, 124002 (2007).

[27] K. G. Arun, B. R. Iyer, B. S. Sathyaprakash, S. Sinha, and C. Van Den Broeck, Phys. Rev. D 76, 104016 (2007).

[28] A.M. Sintes and A. Vecchio, in Rencontres de Moriond:Gravitational Waves and Experimental Gravity, edited by J. Dumarchez (Frontieres, Paris, 2000).

[29] C. Van, Den Broeck, and A.S. Sengupta, Classical Quantum Gravity 24, 155 (2007).

[30] C. Van, Den Broeck, and A.S. Sengupta, Classical Quantum Gravity 24, 1089 (2007).

[31] L. Barack and C. Cutler, Phys. Rev. D 70, 122002 (2004).

[32] L.S. Finn and K.S. Thorne, Phys. Rev. D 62, 124021 (2000).

[33] S. L. Larson, http://www.srl.caltech.edu/ shane/ sensitivity/

[34] F. Pretorius, Phys. Rev. Lett. 95, 121101 (2005).

[35] M. Campanelli, C. O. Lousto, P. Marronetti, and Y. Zlochower, Phys. Rev. Lett. 96, 111101 (2006).

[36] J. G. Baker, J. Centrella, D. I. Choi, M. Koppitz, and J. van Meter, Phys. Rev. Lett. 96, 111102 (2006).

[37] F. Pretorius, Classical Quantum Gravity 23, S529 (2006).

[38] M. Campanelli, C. O. Lousto, and Y. Zlochower, Phys. Rev. D 73, 061501 (2006).

[39] J. G. Baker, J. Centrella, D. I. Choi, M. Koppitz, and J. van Meter, Phys. Rev. D 73, 104002 (2006).

[40] U. Sperhake, Phys. Rev. D 76, 104015 (2007).

[41] B. Bruegmann, J. A. Gonzalez, M. Hannam, S. Husa, U. Sperhake, and W. Tichy, arXiv:gr-qc/0610128.

[42] M. A. Scheel, H. P. Pfeiffer, L. Lindblom, L. E. Kidder, O. Rinne, and S. A. Teukolsky, Phys. Rev. D 74, 104006 (2006).
[43] F. Herrmann, I. Hinder, D. Shoemaker, P. Laguna, and R. A. Matzner, arXiv:gr-qc/0701143.

[44] M. Koppitz, D. Pollney, C. Reisswig, L. Rezzolla, J. Thornburg, P. Diener, and E. Schnetter, Phys. Rev. Lett. 99, 041102 (2007).

[45] S. Husa, M. Hannam, J. A. Gonzalez, U. Sperhake, and B. Brugmann, arXiv:0706.0904.

[46] S. Husa, J. A. Gonzalez, M. Hannam, B. Brugmann, and U. Sperhake, arXiv:0706.0740.

[47] L. Blanchet, Living Rev. Relativity 9, 4 (2006), http:// www.livingreviews.org/lrr-2006-4.

[48] L. Blanchet, Phys. Rev. D 51, 2559 (1995).

[49] L. Blanchet, T. Damour, B. R. Iyer, C. M. Will, and A. G. Wiseman, Phys. Rev. Lett. 74, 3515 (1995).

[50] L. Blanchet, B. R. Iyer, C. M. Will, and A. G. Wiseman, Classical Quantum Gravity 13, 575 (1996).

[51] L. Blanchet, G. Faye, B. R. Iyer, and B. Joguet, Phys. Rev. D 65, 061501 (2002); 71, 129902(E) (2005).

[52] L. Blanchet, T. Damour, G. Esposito-Farese, and B. R. Iyer, Phys. Rev. Lett. 93, 091101 (2004).

[53] S. Nissanke and L. Blanchet, Classical Quantum Gravity 22, 1007 (2005).

[54] K. G. Arun, L. Blanchet, B. R. Iyer, and M. S. S. Qusailah, Classical Quantum Gravity 21, 3771 (2004); 22, 3115(E) (2005).

[55] G. Faye, L. Blanchet, and A. Buonanno, Phys. Rev. D 74, 104033 (2006).

[56] L. Blanchet, A. Buonanno, and G. Faye, Phys. Rev. D 74, 104034 (2006); 75, 049903 (2007).

[57] C. Cutler and E. E. Flanagan, Phys. Rev. D 49, 2658 (1994).

[58] L. S. Finn, Phys. Rev. D 46, 5236 (1992).

[59] C. Cutler and M. Vallisneri, arXiv:0707.2982.

[60] M. Vallisneri, arXiv:gr-qc/0703086.

[61] A. Vecchio (private communication).

[62] S. Babak, A. Pai, and B. F. Schutz (unpublished). 